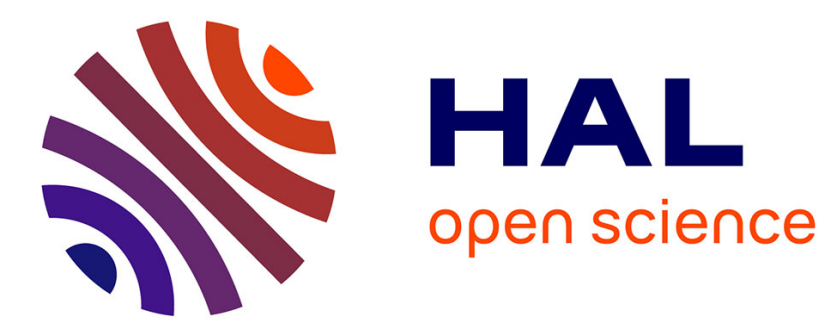

\title{
Theoretical study of SH-wave propagation in periodically-layered piezomagnetic structure
}

Lei Liu, Jinfeng Zhao, Yongdong Pan, Bernard Bonello, Zheng Zhong

\section{To cite this version:}

Lei Liu, Jinfeng Zhao, Yongdong Pan, Bernard Bonello, Zheng Zhong. Theoretical study of SHwave propagation in periodically-layered piezomagnetic structure. International Journal of Mechanical Sciences, 2014, 85, pp.45-54. 10.1016/j.ijmecsci.2014.04.028 . hal-01233803

\section{HAL Id: hal-01233803 https://hal.science/hal-01233803}

Submitted on 30 Apr 2020

HAL is a multi-disciplinary open access archive for the deposit and dissemination of scientific research documents, whether they are published or not. The documents may come from teaching and research institutions in France or abroad, or from public or private research centers.
L'archive ouverte pluridisciplinaire HAL, est destinée au dépôt et à la diffusion de documents scientifiques de niveau recherche, publiés ou non, émanant des établissements d'enseignement et de recherche français ou étrangers, des laboratoires publics ou privés.

\section{(c)(1)}

Distributed under a Creative Commons Attribution| 4.0 International License 


\title{
Theoretical study of SH-wave propagation in periodically-layered piezomagnetic structure
}

\author{
Lei LIU ${ }^{1}$, Jinfeng ZHAO², Yongdong PAN ${ }^{1, a)}$, \\ Bernard BONELLO ${ }^{2}$, and Zheng ZHONG ${ }^{1, b)}$ \\ ${ }^{1}$ School of Aerospace Engineering and Applied Mechanics, Tongji University, Shanghai 200092, P. R. China \\ ${ }^{2}$ Institute of NanoSciences, University Pierre et Marie Curie, Paris 75252, France
}

\begin{abstract}
In this paper, the propagation of SH (shear horizontal) wave in the periodically-layered piezomagnetic structure is studied theoretically. Both the dispersion equation and transmission coefficients are derived to reveal the wave behavior when the piezomagnetism is ignored or the magnetic circuit is closed and open. The zero-order mode of the piezomagnetism-ignored single layer is not dispersive and every higher order mode is dispersive with a cut-off frequency. Same features are found for the closed and open cases, except that the zero-order mode of the latter case is dispersive. The pass bands of the piezomagnetism-ignored periodically-layered structure appear when the normalized frequency is an even integer under the normal incidence, and new stop bands will appear from the pass bands when the incident angle increases. Same features are observed for the band gaps of the magnetically closed and open cases, except that the zero-order mode of the latter case is dispersive.
\end{abstract}

Keywords: SH wave; periodic structure; dispersion; band gaps; piezomagnetism

\section{INTRODUCTION}

Periodically-layered piezomagnetic structures are class of one-dimensional phononic crystal (PC) whose material parameters are periodically arranged in space. Under certain conditions, these hetero structures exhibit many amazing properties including band gaps, negative refraction of elastic waves etc. All these properties make these PCs very promising to potential applications, such as wave filters, sonic lenses and so on.

The PCs have received extensive attention since they were investigated for the first time by Kushwaha et al. [1] twenty years ago. In this seminal work, it was established that the three-dimensional (3D) PCs have many amazing properties which cannot exist in nature; later the two-dimensional (2D) and one-dimensional (1D) PCs were analyzed, and it was shown that both have identical features as well [2-4]. These features may concern any type of waves, including the longitudinal wave, transverse wave [3], P-SV waves [4], etc.

Recently, both piezoelectric(PE) and piezomagnetic(PM) materials were introduced to the PC to create special smart structures [5-9].The PCs with PE material attracted extensive attention, and the propagation of SH wave in such a type of PCs was discussed recently [10]. Simultaneously, as much as the piezoelectricity, the piezomagnetism which is the inverse of the magnetostrictive effect, have also attracted much attention. But most of the researches on this topic are focused on the magnetoelectric conversion in composite structures made of PE and PM materials [11,12]. However, the interaction between the wave propagation and the PCs with PM materials is still not enough understood. One knows that the piezomagnetism has to be included in the constitutive equation of the material, and thus that it plays a key role in the monitoring of the band gaps in such PCs. Although the propagation of the relatively complex P-SV wave in these systems has been analyzed, the relationship between the band gaps and the incident angle is still imperfectly known $[9,13]$, even when the piezomagnetism is ignored $[2,10]$.

In the mid of the nineties, Auld et al. [14] have paid attention to the propagation of shear horizontal waves (SH) in 1D PC. They have shown that the opening of a band gaps occurs even in this relatively simple system. Later, Qian et al.[6] in 2004 analyzed the propagation of SH waves in 1D PC made of a PE material and polymeric ceramics. Nie G Q et al.[15] in

\footnotetext{
a) Tel. 86-021-6598-3797, Email address: ypan@tongji.edu.cn.

b) Tel. 86-021-6598-3998, Email address: zhongk@tongji.edu.cn.
} 
2007 studied the propagation of SH wave in two layered PE/PM coupled plates, and pointed out the influences both of the PE material properties and of the ratios of the thicknesses of the PE layer to the PM layer on the propagation behaviors. More recently, Liu J X et al. [7] in 2010 analyzed the propagation of SH waves in PE-PM periodically layered structures. They have computed the dispersion relations for when the direction of propagation is either normal or parallel to the interface. Although the band gaps of the PE-PM PCs have been identified in these works [7,9,13], their relationship with the incident angle and the influence of the piezomagnetism to their location and magnitude have not been investigated to date. In a previous paper, we have analyzed the propagation of SH waves both in a single PE layer and in PEPCs [10]. However the PM material has its own properties which are totally different from the ones of the PE material and the knowledge of the wave propagation in the PM PCs is still lacking.

Measuring the transmission coefficients is the straightest way to experimentally determine the band gaps for a direct comparison with the computation of the dispersion relation. It is therefore of great importance to study them. One can also compute the transmission coefficients but, due to the inherent instability of the transfer matrix, this could be particularly challenging $[16,17]$. Global transfer matrix is one of the most useful methods to calculate the transmission coefficients, but the computing time may increase quickly with the increase of PC's thickness. Meanwhile, it needs being properly implemented and it faces more challenges at high frequency [18]. Although the periodic PE and PM materials were recently considered as layered structures, the transmission coefficients are still difficult to be obtained $[9,13]$.

The magnetic boundary conditions for the PM material may significantly affect the propagation of the wave due to the introduction of piezomagnetism. This phenomenon has been theoretically analyzed for the P-SV wave and the relationship between the phase velocity and the magnetic boundary condition of the PE/PM bi-material plate has been established numerically [19]. However, little attention has been paid to the propagation of SH wave under different magnetic boundary conditions.

In this article, the SH wave propagation in the periodically-layered PM structure is studied. We first describe the physical model and the basic formulation. The dispersion relation of a single PM layer and of a layered periodic structure will then be derived for different magnetic boundary conditions from which the corresponding transmission coefficients are obtained. Numerical results are given to demonstrate the characteristics of the SH wave propagation in both single and periodic layer of PM materials.

\section{PHYSICAL MODEL AND BASIC FORMULATION}

\subsection{Physical model}

Consider a steady anti-plane shear $(\mathrm{SH})$ wave propagating in the $x-y$ plane with its displacement along $z$-direction. The SH wave propagates from a semi-infinite material (I)through a transversely isotropic elastic material (A) or a transversely isotropic PM material (B) to another semi-infinite material (II) with the incident angle $\theta_{0}$ as shown in Fig.1(a). We suppose that media I and II are made of the same transversely isotropic elastic material with density $\rho_{\mathrm{I}}$. We further suppose that the single layer of material A or B has the density $\rho$ and the thickness $h$. The PM material is magnetized along the $z$-coordinate. Moreover, we consider a periodic structure on which SH wave propagate (Fig. 1(b)). The periodic structure is made by alternating layer A (density $\rho_{\mathrm{A}}$ and thickness $h_{\mathrm{A}}$ ) and layer B (density $\rho_{\mathrm{B}}$ and thickness $h_{\mathrm{B}}$ ) as shown in Fig. 1(a). The unit cell of the periodic structure has a thickness $d=h_{\mathrm{A}}+h_{\mathrm{B}}$, and the number of cells is $N$, so that the total thickness is $L=N d$. Numbers 1 , 2, 3...in Fig. 1(b) indicate the interfaces between the different layers.

\subsection{Basic formulation}

Since we are dealing with the propagation of elastic waves in the linear regime, only small dynamic perturbations around an equilibrium state due to the incidence of the SH wave, is considered. The constitutive equations of the transversely isotropic PM or elastic material under conditions of small wave perturbations, could be written as $8,9,13,15,17$

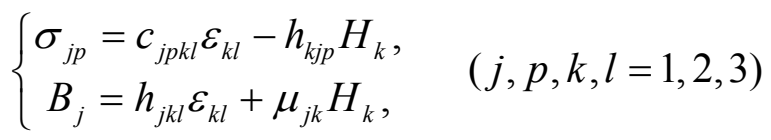

with the dynamic stress $\sigma_{j p}$, the dynamic strain $\varepsilon_{k l}$, the dynamic magnetic field intensity $H_{k}$, the dynamic magnetic induction $B_{j}$, the elastic constants $c_{j p k l}$, the magnetic permeability $\mu_{k}$, the PM constants $h_{j k l}$. Subscript 1, 2 and 3 stand for $x$-, $y$ - and $z$ coordinates respectively. The corresponding constitutive equations for the layers I, A, B and II can be found just by replacing the material properties of Eq. (1) with their corresponding material constants. The elastic constants $c_{j p k l}^{\mathrm{A}}$, the permeability

constants $\mu_{j k}^{\mathrm{A}}$, and the PM constants $h_{j k l}^{\mathrm{A}}$ are for the layer A. The superscript A of the constants can be replaced by B and I to 
LIU, ZHAO, PAN, BONELLO, ZHONG

denote the corresponding material properties of the layers B and I. The general form of the dynamic differential equations for the transversely isotropic PM or elastic material are

$$
\rho \partial^{2} u_{j} / \partial t^{2}=\sigma_{j p, p}, \quad B_{j, j}=0, \quad(j, p=1,2,3)
$$

where $u_{j}$ is the dynamic displacement in the $j$ th direction, a comma followed by a subscript $p$ means space differentiation with respect to the $p$-coordinate. The dynamic strain tensor and the intensity of the magnetic field can be expressed as

$$
\varepsilon_{k l}=\left(u_{k, l}+u_{l, k}\right) / 2, \quad H_{k}=-\psi_{, k}, \quad(k, l=1,2,3)
$$

where $\psi$ is the magnetic potential function. All the following variables are related to the dynamic part introduced by the wave excitation around the equilibrium state. The displacement $u_{1}, u_{2}$ and $u_{3}$ and the magnetic potential function $\psi$ are supposed to have solutions in the form

$$
u_{1}=u_{2}=0, \quad u_{3}=U_{3}(y) \exp \left[i \omega\left(t-s_{x} x\right)\right], \quad \psi=\psi(y) \exp \left[i \omega\left(t-s_{x} x\right)\right],
$$

where the angular frequency is $\omega$ (frequency $f=\omega / 2 \pi$ ), the slowness along $x$-coordinate is $s_{x}$, the time is $t$ and the unit of imaginary number is $i . U_{3}(y)$ and $\Psi(y)$ stand for the amplitudes of $u_{3}$ and $\Psi$ respectively. Substituting Eq. (4) into Eq. (3), the strain tensor and the magnetic field intensity are derived as

$$
\left\{\begin{array}{l}
\varepsilon_{11}=\varepsilon_{22}=\varepsilon_{33}=\varepsilon_{12}=0, \quad 2 \varepsilon_{23}=u_{3,1}, \quad 2 \varepsilon_{31}=u_{3,1}, \\
H_{1}=-\psi_{, 1}, \quad H_{2}=-\psi_{, 2}, \quad H_{3}=0 .
\end{array}\right.
$$

Substituting Eq. (5) into Eq. (1), the stress tensor and the magnetic induction can be written as

$$
\begin{aligned}
& \left\{\begin{array}{lcl}
\sigma_{11}=0, & \sigma_{22}=0, & \sigma_{33}=0, \\
\sigma_{23}=c_{44} u_{3,2}+h_{24} \psi_{, 2}, & \sigma_{31}=c_{55} u_{3,1}+h_{15} \psi_{, 1}, & \sigma_{12}=0,
\end{array}\right. \\
& B_{1}=h_{15} u_{3,1}-\mu_{11} \psi_{, 1}, \quad B_{2}=h_{24} u_{3,2}-\mu_{22} \psi_{, 2}, \quad B_{3}=0 .
\end{aligned}
$$

Since we only consider transversely isotropic PM or elastic materials, $\mathrm{h}_{15}=\mathrm{h}_{24}$ in the coupling matrix. The partial differential equations could therefore be obtained from Eqs. (6) as

$$
u_{3,2}=\left(\mu_{11} \sigma_{23}+h_{15} B_{2}\right) / \Delta, \quad \varphi_{, 2}=\left(h_{15} \sigma_{23}-c_{44} B_{2}\right) / \Delta,
$$

where $\Delta=c_{44} \mu_{11}+h_{15}^{2}$. Substituting Eqs. (6) into Eq. (2), we obtain the partial differential equations

$$
\sigma_{23,2}=\omega^{2}\left[\left(-\rho+c_{44} s_{x}^{2}\right) u_{3}+h_{15} s_{x}^{2} \psi\right], \quad B_{2,2}=\omega^{2}\left(h_{15} s_{x}^{2} u_{3}-\mu_{11} s_{x}^{2} \psi\right) .
$$

If moreover we define the vector $\vec{\eta}=\left[\begin{array}{llll}v_{3} & \chi & \sigma_{23} & B_{2}\end{array}\right]^{\prime}$ where $v_{3}=\partial u_{3} / \partial t$ and $\chi=\partial \psi / \partial t$, the state-vector form of equations could be derived from Eqs. (7) and (8) as

$$
\vec{\eta}_{, 2}=i \omega \Pi \vec{\eta}
$$

where the system matrix $\Pi$ is

$$
\Pi=\left[\begin{array}{cccc}
0 & 0 & \mu_{11} \Delta^{-1} & h_{15} \Delta^{-1} \\
0 & 0 & h_{15} \Delta^{-1} & -c_{44} \Delta^{-1} \\
\rho-c_{44} s_{x}^{2} & -h_{15} s_{x}^{2} & 0 & 0 \\
-h_{15} s_{x}^{2} & \mu_{11} s_{x}^{2} & 0 & 0
\end{array}\right] .
$$

Here Eqs. (1)-(9)can be applied to the materials of the layers I, A, B and II, if considering their respective physical properties. Waves propagating in the periodic structure satisfy the Bloch's law as

$$
\vec{\eta}_{2 n+1}=e^{i \Lambda d} \vec{\eta}_{2 n-1}
$$

where $\Lambda$ is the periodic wave number. 


\section{CONTINUITY CONDITIONS}

For the problem described in Fig. 1(b), the velocity $v_{3}$, the stress component $\sigma_{23}$, the parameter $\chi$ and the magnetic induction $B$ are continuous along the interfaces. These boundary conditions lead to the following relations

$$
v_{3}^{I}=v_{3}^{A}, \quad \sigma_{23}^{I}=\sigma_{23}^{A}, \quad \chi^{I}=\chi^{A}, \quad B_{2}^{I}=B_{2}^{A},
$$

for the interface 1,

$$
v_{3}^{A}=v_{3}^{B}, \quad \sigma_{23}^{A}=\sigma_{23}^{B}, \quad \chi^{A}=\chi^{B}, \quad B_{2}^{A}=B_{2}^{B},
$$

for the interfaces $2,3 \ldots 2 N$ and

$$
v_{3}^{B}=v_{3}^{I I}, \quad \sigma_{23}^{B}=\sigma_{23}^{I I}, \quad \chi^{B}=\chi^{I I}, \quad B_{2}^{B}=B_{2}^{I I},
$$

for the interface $2 N+1$.

For the magnetically open boundary condition of the PM materials, the magnetic potential function $\psi$ must be zero along interfaces $1,2,3 \ldots 2 N+1$. Under this hypothesis, the velocity $v_{3}$ and stress $\sigma_{23}$ still satisfy the Bloch's law

$$
\left(\begin{array}{c}
v_{3} \\
\sigma_{23}
\end{array}\right)_{2 n+1}=e^{i \Lambda d}\left(\begin{array}{c}
v_{3} \\
\sigma_{23}
\end{array}\right)_{2 n-1} .
$$

Meanwhile the velocity $v_{3}$ and the stress component $\sigma_{23}$ also satisfy Eqs. (11). Similar procedures can be applied to analyze the continuity conditions for the problem described in Fig. 1(a).

\section{SOLUTION OF DISPERSION}

The general analytical solutions of Eqs.(7) and (8) have been given by Qian et al. ${ }^{6}$ while using a different approach, which could be summarized as

$$
\left\{\begin{array}{l}
u_{3}=\left(C_{1}^{+} e^{i \omega s_{y} y}+C_{1}^{-} e^{-i \omega s_{y} y}\right) e^{i \omega\left(t-s_{x} x\right)} \\
\psi=\left[h_{15} \mu_{11}^{-1}\left(C_{1}^{+} e^{i \omega s_{y} y}+C_{1}^{-} e^{-i \omega s_{y} y}\right)+\left(C_{2}^{+} e^{\omega s_{x} y}+C_{2}^{-} e^{-\omega s_{x} y}\right)\right] e^{i \omega\left(t-s_{x} x\right)}, \\
\sigma_{23}=\omega\left[i s_{y} \bar{c}_{44}\left(C_{1}^{+} e^{i \omega s_{y} y}-C_{1}^{-} e^{-i \omega s_{y} y}\right)+h_{15} s_{x}\left(C_{2}^{+} e^{\omega s_{x} y}-C_{2}^{-} e^{-\omega s_{x} y}\right)\right] e^{i \omega\left(t-s_{x} x\right)}, \\
B_{2}=-\omega \mu_{11} s_{x}\left(C_{2}^{+} e^{\omega s_{x} y}-C_{2}^{-} e^{-\omega s_{x} y}\right) e^{i \omega\left(t-s_{x} x\right)}
\end{array}\right.
$$

with $s_{y}=\sqrt{\rho / \bar{c}_{44}-s_{x}^{2}}, \quad \bar{c}_{44}=c_{44}+\left(h_{15}\right)^{2} / \mu_{11}$, where $C_{1}^{+}, C_{1}^{-}, C_{2}^{+}$and $C_{2}^{-}$are unknown coefficients, $s_{y}$ is the slowness along $y$-axis, $\bar{c}_{44}$ is the piezomagnetically stiffened elastic constant. If the PM constant $h_{15}$ approaches zero, the PM effect can be ignored for the SH propagation, and Eq. (13a) will become

$$
\left\{\begin{array}{l}
u_{3}=\left(C_{1}^{+} e^{i \omega \hat{s}_{y} y}+C_{1}^{-} e^{-i \omega \hat{s}_{y} y}\right) e^{i \omega\left(t-s_{x} x\right)} \\
\psi=\left(C_{2}^{+} e^{\omega s_{x} y}+C_{2}^{-} e^{-\omega s_{x} y}\right) e^{i \omega\left(t-s_{x} x\right)} \\
\sigma_{23}=\omega i \hat{s}_{y} c_{44}\left(C_{1}^{+} e^{i \omega \hat{s}_{y} y}-C_{1}^{-} e^{-i \omega \hat{s}_{y} y}\right) e^{i \omega\left(t-s_{x} x\right)} \\
B_{2}=-\omega \mu_{11} s_{x}\left(C_{2}^{+} e^{\omega s_{x} y}-C_{2}^{-} e^{-\omega s_{x} y}\right) e^{i \omega\left(t-s_{x} x\right)}
\end{array}\right.
$$

with $\hat{s}_{y}=\sqrt{\rho / c_{44}-s_{x}^{2}}$. These are the general solutions for the magnetically closed circuit boundary condition if slowness $\hat{S}_{y}$ is replaced by $s_{y}$. 
LIU, ZHAO, PAN, BONELLO, ZHONG

For the magnetically open circuit boundary condition, the general solution of displacement $u_{3}$ takes the same form as that in Eqs. (13a) and (13b), but the solutions for the potential function $\psi$ and stress $\sigma_{23}$ are different. They are found to be

$$
\left\{\begin{array}{l}
\psi=\frac{h_{15}}{\mu_{11}}\left[\begin{array}{l}
\left(C_{1}^{+} e^{i \omega s_{y} y}+C_{1}^{-} e^{-i \omega s_{y} y}\right)+ \\
C_{1}^{+}\left(a_{11} e^{\omega s_{x} y}+a_{21} e^{-\omega s_{x} y}\right)+C_{1}^{-}\left(a_{12} e^{\omega s_{x} y}+a_{22} e^{-\omega s_{x} y}\right)
\end{array}\right] e^{i \omega\left(t-s_{x} x\right)}, \\
\sigma_{23}=\omega\left\{\begin{array}{l}
i s_{y} \bar{c}_{44}\left(C_{1}^{+} e^{i \omega s_{y} y}-C_{1}^{-} e^{-i \omega s_{y} y}\right) \\
+h_{15}^{2} \mu_{11}^{-1} s_{x}\left[C_{1}^{+}\left(a_{11} e^{\omega s_{x} y}-a_{21} e^{-\omega s_{x} y}\right)+C_{1}^{-}\left(a_{12} e^{\omega s_{x} y}-a_{22} e^{-\omega s_{x} y}\right)\right]
\end{array}\right\} e^{i \omega\left(t-s_{x} x\right)},
\end{array}\right.
$$

with

$$
\left\{\begin{array}{l}
{\left[a_{11}, a_{12}, a_{21}, a_{22}\right]=\left[e^{-\delta_{x}}-e^{i \delta_{y}}, e^{-\delta_{x}}-e^{-i \delta_{y}}, e^{i \delta_{y}}-e^{\delta_{x}}, e^{-i \delta_{y}}-e^{\delta_{x}}\right] / \Delta_{x}} \\
\delta_{x}=\omega s_{x} h, \quad \delta_{y}=\omega s_{y} h, \quad \Delta_{x}=e^{\delta_{x}}-e^{-\delta_{x}}
\end{array}\right.
$$

If the PM effect is ignored, Eq. (14a) can be simplified into

$$
\left\{\begin{array}{l}
\psi=0 \\
\sigma_{23}=\omega i \hat{s}_{y} c_{44}\left(C_{1}^{+} e^{i \omega \hat{s}_{y} y}-C_{1}^{-} e^{-i \omega \hat{s}_{y} y}\right) e^{i \omega\left(t-s_{x} x\right)}
\end{array}\right.
$$

After comparison of stress $\sigma_{23}$ in Eqs. (13) and (14), it can be found that the influence of the magnetic boundary condition (closed or open circuit) appears only when the PM effect is not ignored. The analytical solutions described in Eqs.(13) and (14) are suitable for the layers I, A, B and II just by introducing there in the corresponding material properties.

\subsection{Single layer}

Consider the SH wave propagation in a single layer shown in Fig. 1(a) and let media I and II be vacuum. The magnetic induction $B_{2}$ and stress component $\sigma_{23}$ satisfy

$$
\sigma_{23}=0, \quad B_{2}=0
$$

at $y=0$ and $y=h$ for the magnetically closed circuit boundary. Substituting Eq. (15) into Eq. (13a), the coefficients $C_{2}^{+}$and $C_{2}^{-}$shall be zero. The expression of $\sigma_{23}$ in Eq. (13a) can be rewritten in terms of cosine and sine functions, so that the SH wave's dispersion equation could be derived as

$$
s_{x}= \pm \sqrt{\frac{\rho}{\bar{c}_{44}}-\left(\frac{m}{2 f h}\right)^{2}},(m=0,1,2,3 \ldots)
$$

where $m$ is the mode number. If the PM effect is neglected, the elastic constant $\bar{C}_{44}$ should be replaced by $C_{44}$ in Eq. (16). If the magnetically open circuit boundary is considered, the stress component $\sigma_{23}$ and the magnetic potential function $\psi$ satisfy

$$
\sigma_{23}=0, \quad \psi=0
$$

at $y=0$ and $y=h$. Substituting Eq. (17) into Eq. (14a), the SH wave's dispersion equation becomes

$$
\left[i s_{y}-\frac{h_{15}^{2} s_{x}}{\bar{c}_{44} \mu_{11}}\left(a_{12}-a_{22}\right)\right]^{2}-e^{-2 i s_{y} h}\left[i s_{y}+\frac{h_{15}^{2} s_{x}}{\bar{c}_{44} \mu_{11}}\left(a_{11}-a_{21}\right)\right]^{2}=0 \text {. }
$$

If the PM effect can be neglected, Eq. (18) takes the same form as Eq. (16) with $\bar{c}_{44}=c_{44}$. As the coefficients $a_{11}, a_{12}, a_{21}$ and $a_{22}$ depend on the slowness $s_{x}$ and $s_{y}$, it's challenging to decouple Eq. (18), and numerical calculations are needed for further analysis. 


\subsection{Periodic structure}

For SH wave propagating in the periodic structure displayed in Fig. 1(b), the $4 \times 4$ transfer matrices $M$ could be derived for each layer A and B from the system matrix $\Psi$ in Eq. (9) as [20]

$$
\begin{cases}\vec{\eta}_{2 n}=M^{\mathrm{A}} \vec{\eta}_{2 n-1}, & \vec{\eta}_{2 n+1}=M^{\mathrm{B}} \vec{\eta}_{2 n}, \\ M^{\alpha}=X_{\alpha} \Theta_{\alpha} X_{\alpha}^{-1}, & \Theta_{\alpha}=\operatorname{diag}\left[\exp \left(i \omega \lambda_{\beta}^{\alpha} h_{\alpha}\right)\right], \quad(\alpha=\mathrm{A}, \mathrm{B} ; \beta=1,2,3,4)\end{cases}
$$

where the $\lambda_{\beta}^{\alpha}$ and $X_{\alpha}$ are respectively the eigen value and eigenvector of the system matrix $\Pi_{\alpha}$ for the layer $\alpha$. If the general velocity vector $\vec{v}$ and stress vector $\vec{\sigma}$ are set as $\vec{v}=\left[\begin{array}{ll}v_{3} & \psi\end{array}\right]^{\prime}$ and $\vec{\sigma}=\left[\begin{array}{ll}\sigma_{23} & B_{2}\end{array}\right]^{\prime}$ respectively, the following $4 \times 4$ compliance matrices could be derived from Eq. (19) as

$$
\left[\begin{array}{l}
\vec{v}_{2 n-1} \\
\vec{v}_{2 n}
\end{array}\right]=S^{\mathrm{A}}\left[\begin{array}{l}
\vec{\sigma}_{2 n-1} \\
\vec{\sigma}_{2 n}
\end{array}\right],\left[\begin{array}{l}
\vec{v}_{2 n} \\
\vec{v}_{2 n+1}
\end{array}\right]=S^{\mathrm{B}}\left[\begin{array}{l}
\vec{\sigma}_{2 n} \\
\vec{\sigma}_{2 n+1}
\end{array}\right],
$$

where the compliance matrix reads

$$
S^{\alpha}=\left[\begin{array}{cc}
-\left(M_{21}^{\alpha}\right)^{-1} M_{22}^{\alpha} & \left(M_{21}^{\alpha}\right)^{-1} \\
M_{12}^{\alpha}-M_{11}^{\alpha}\left(M_{21}^{\alpha}\right)^{-1} M_{22}^{\alpha} & M_{11}^{\alpha}\left(M_{21}^{\alpha}\right)^{-1}
\end{array}\right],(\alpha=\mathrm{A} \text { or B })
$$

In Eq. (20b) $M_{j p}^{\alpha}(j, p=1,2)$ is the $j$ th row and $p$ th column $2 \times 2$ sub-matrix of $\mathrm{M}^{\alpha}$. The cell compliance matrix $\mathrm{S}^{\mathrm{C}}$ of the periodic structure can be derived from Eq. (20) as

$$
S^{\mathrm{C}}=\left[\begin{array}{cc}
S_{11}^{\mathrm{A}}+S_{12}^{\mathrm{A}}\left(S_{11}^{\mathrm{B}}-S_{22}^{\mathrm{A}}\right)^{-1} S_{21}^{\mathrm{A}} & -S_{12}^{\mathrm{A}}\left(S_{11}^{\mathrm{B}}-S_{22}^{\mathrm{A}}\right)^{-1} S_{12}^{\mathrm{B}} \\
S_{21}^{\mathrm{B}}\left(S_{11}^{\mathrm{B}}-S_{22}^{\mathrm{A}}\right)^{-1} S_{21}^{\mathrm{A}} & S_{22}^{\mathrm{B}}-S_{21}^{\mathrm{B}}\left(S_{11}^{\mathrm{B}}-S_{22}^{\mathrm{A}}\right)^{-1} S_{12}^{\mathrm{B}}
\end{array}\right],
$$

with

$$
\left[\begin{array}{l}
\vec{v}_{2 n-1} \\
\vec{v}_{2 n+1}
\end{array}\right]=S^{\mathrm{C}}\left[\begin{array}{l}
\vec{\sigma}_{2 n-1} \\
\vec{\sigma}_{2 n+1}
\end{array}\right]
$$

The $S_{j p}^{\alpha}(j, p=1,2)$ is the $j$ th row and $p$ th column $2 \times 2$ sub-matrix of $\mathrm{S}^{\alpha}$. After the substitution of Eq. (21) into Eq. (10), the dispersion equation of the periodic structure is obtained as

$$
\left|S_{12}^{\mathrm{C}}\left(e^{i \Lambda d}\right)^{2}+\left(S_{11}^{\mathrm{C}}-S_{22}^{\mathrm{C}}\right) e^{i \Lambda d}-S_{21}^{\mathrm{C}}\right|=0 .
$$

When considering the magnetically open circuit boundary, the $2 \times 2$ transfer matrices for each layer of the periodic structure can be derived as

$$
\left[\begin{array}{c}
v_{3} \\
\sigma_{23}
\end{array}\right]_{2 n}=M^{\mathrm{A}}\left[\begin{array}{c}
v_{3} \\
\sigma_{23}
\end{array}\right]_{2 n-1}, M^{\mathrm{A}}=\left[\begin{array}{cc}
\cos \left(\delta_{y}^{\mathrm{A}}\right) & i \sin \left(\delta_{y}^{\mathrm{A}}\right) /\left(Z_{y}^{\mathrm{A}}\right) \\
i Z_{y}^{\mathrm{A}} \sin \left(\delta_{y}^{\mathrm{A}}\right) & \cos \left(\delta_{y}^{\mathrm{A}}\right)
\end{array}\right],
$$

for the layer A with $\delta_{y}^{\mathrm{A}}=\omega h_{\mathrm{A}} s_{y}^{\mathrm{A}}, Z_{y}^{\mathrm{A}}=\rho_{\mathrm{A}}\left(c_{s h}^{\mathrm{A}}\right)^{2} s_{y}^{\mathrm{A}}, s_{y}^{\mathrm{A}}=\sqrt{1 /\left(c_{s h}^{\mathrm{A}}\right)^{2}-s_{x}^{2}}, c_{s h}^{\mathrm{A}}=\sqrt{c_{44}^{\mathrm{A}} / \rho_{\mathrm{A}}}$, and

$$
\left[\begin{array}{c}
v_{3} \\
\sigma_{23}
\end{array}\right]_{2 n+1}=M^{\mathrm{B}}\left[\begin{array}{c}
v_{3} \\
\sigma_{23}
\end{array}\right]_{2 n}, M^{\mathrm{B}}=\left[\begin{array}{cc}
\tau e^{i \delta_{y}^{\mathrm{B}}}+\varsigma e^{-i \delta_{y}^{\mathrm{B}}} & 2 i \sin \left(\delta_{y}^{\mathrm{B}}\right) \\
\tau^{2} e^{i \delta_{y}^{\mathrm{B}}}-\varsigma^{2} e^{-i \delta_{y}^{\mathrm{B}}} & \tau e^{i \delta_{y}^{\mathrm{B}}}+\varsigma e^{-i \delta_{y}^{\mathrm{B}}}
\end{array}\right] \frac{1}{\tau+\varsigma},
$$

for the layer B with 


$$
\left\{\begin{array}{llll}
\tau=Z_{y}^{\mathrm{B}}+i Z_{x}^{\mathrm{B}}\left(a_{12}-a_{22}\right), & \varsigma=Z_{y}^{\mathrm{B}}-i Z_{x}^{\mathrm{B}}\left(a_{11}-a_{21}\right), & \delta_{x}^{\mathrm{B}}=\omega h_{\mathrm{B}} s_{x}, & \delta_{y}^{\mathrm{B}}=\omega h_{\mathrm{B}} s_{y}^{\mathrm{B}}, \\
s_{y}^{\mathrm{B}}=\sqrt{1 /\left(\bar{c}_{s h}^{\mathrm{B}}\right)^{2}-s_{x}^{2}}, & Z_{x}^{\mathrm{B}}=s_{x}\left(h_{15}^{\mathrm{B}}\right)^{2} / \mu_{11}^{\mathrm{B}}, & Z_{y}^{\mathrm{B}}=\rho_{\mathrm{B}}\left(\bar{c}_{s h}^{\mathrm{B}}\right)^{2} s_{y}^{\mathrm{B}}, & \bar{c}_{s h}^{\mathrm{B}}=\sqrt{\bar{c}_{44}^{\mathrm{B}} / \rho_{\mathrm{B}}} .
\end{array}\right.
$$

If the $\mathrm{PM}$ effect is ignored, the transfer matrix $\mathrm{M}^{\mathrm{B}}$ takes the same form as $\mathrm{M}^{\mathrm{A}}$. The cell transfer matrix $\mathrm{M}^{\mathrm{C}}$ of the periodic structure satisfies

$$
\left[\begin{array}{c}
v_{3} \\
\sigma_{23}
\end{array}\right]_{2 n+1}=M^{\mathrm{C}}\left[\begin{array}{c}
v_{3} \\
\sigma_{23}
\end{array}\right]_{2 n-1}, M^{\mathrm{C}}=M^{\mathrm{B}} M^{\mathrm{A}} .
$$

After the substitution of Eq. (25) into Eq. (12), the dispersion equation of the periodic structure is expressed as

$$
\left|M^{\mathrm{B}} M^{\mathrm{A}}-e^{i \Lambda d}\right|=0 \text {. }
$$

\section{SOLUTION OF TRANSMISSION COEFFICIENTS}

For the problem described in Fig. 1(b), the displacement for the semi-infinite layers I and II can be respectively expressed as

$$
u_{3}^{\mathrm{I}}=\left(a^{+} e^{i \omega s_{y}^{\mathrm{I} y}}+a^{-} e^{-i \omega s_{y}^{\mathrm{I} y}}\right) e^{i \omega\left(t-s_{x} x\right)}, \quad u_{3}^{\mathrm{II}}=b^{+} e^{i \omega s_{y}^{\mathrm{I}}(y-L)} e^{i \omega\left(t-s_{x} x\right)},
$$

where $s_{y}^{\mathrm{I}}=\sqrt{1 /\left(c_{s h}^{\mathrm{I}}\right)^{2}-s_{x}^{2}}, c_{s h}^{\mathrm{I}}=\sqrt{c_{44}^{\mathrm{I}} / \rho_{\mathrm{I}}} \cdot a^{+}, a^{-}$and $b^{+}$are unknown coefficients. The magnetic potential function for layers I and II are assumed to read respectively

$$
\psi_{\mathrm{I}}(x, y, t)=C_{\mathrm{I}} e^{\omega s_{x} y} e^{i \omega\left(t-s_{x} x\right)}, \quad \psi_{\mathrm{II}}(x, y, t)=C_{\mathrm{II}} e^{-\omega s_{x}(y-L)} e^{i \omega\left(t-s_{x} x\right)},
$$

where $C_{\mathrm{I}}$ and $C_{\mathrm{II}}$ are unknown coefficients. The vector $\vec{\eta}$ along interfaces 1 and $2 N+1$ could then be derived as

$$
\begin{cases}\vec{\eta}_{1}=i \omega a^{+} W \vec{\zeta}_{\mathrm{I}}, & \vec{\eta}_{2 N+1}=i \omega a^{+} W \vec{\zeta}_{\mathrm{II}}, \\
\vec{\zeta}_{\mathrm{I}}=\left[\begin{array}{llll}
1 & \vartheta & C_{\mathrm{I}} / a^{+} & 0
\end{array}\right], & \vec{\zeta}_{\mathrm{II}}=\left[\begin{array}{llll}
\xi & 0 & 0 & C_{\mathrm{II}} / a^{+}
\end{array}\right],\end{cases}
$$

where the transmission coefficient $\xi=b^{+} / a^{+}$, the transmissivity $\mathrm{T}=\left|b^{+} / a^{+}\right|^{2}$, the reflective coefficient $\vartheta=a^{-} / a^{+}$, and the reflectivity $\mathrm{R}=\left|a^{-} / a^{+}\right|^{2}$. The matrix $W$ is expressed as

$$
W=\left[\begin{array}{cccc}
1 & 1 & 0 & 0 \\
0 & 0 & 1 & 1 \\
s_{\mathrm{I}} c_{44}^{\mathrm{I}} & -S_{\mathrm{I}} c_{44}^{\mathrm{I}} & 0 & 0 \\
0 & 0 & i s_{x} \mu_{11}^{\mathrm{I}} & -i s_{x} \mu_{11}^{\mathrm{I}}
\end{array}\right] .
$$

If the continuity conditions are considered, the total compliance matrix $\mathrm{S}^{\mathrm{T}}$ of the periodic structure could be derived by a recursive algorithm [21], and it is found as

$$
\left[\begin{array}{l}
\vec{v}_{1} \\
\vec{v}_{2 N+1}
\end{array}\right]=S^{\mathrm{T}}\left[\begin{array}{l}
\vec{\sigma}_{1} \\
\vec{\sigma}_{2 N+1}
\end{array}\right], S^{\mathrm{T}}=\left[\begin{array}{ll}
S_{11}^{\mathrm{T}} & S_{12}^{\mathrm{T}} \\
S_{21}^{\mathrm{T}} & S_{22}^{\mathrm{T}}
\end{array}\right] .
$$

If Eq. (30) is substituted into Eq. (29), one finds

$$
\vec{\zeta}_{\mathrm{I}}=W^{-1} M^{S} W \vec{\zeta}_{\mathrm{II}}, M^{S}=\left[\begin{array}{cc}
S_{22}^{\mathrm{T}}\left(S_{12}^{\mathrm{T}}\right)^{-1} & S_{21}^{\mathrm{T}}-S_{22}^{\mathrm{T}}\left(S_{12}^{\mathrm{T}}\right)^{-1} S_{11}^{\mathrm{T}} \\
\left(S_{12}^{\mathrm{T}}\right)^{-1} & -\left(S_{12}^{\mathrm{T}}\right)^{-1} S_{11}^{\mathrm{T}}
\end{array}\right] .
$$

The transmission coefficients can be calculated using Eq. (31). If the magnetically closed circuit boundary is considered, the total transfer matrix $\mathrm{M}^{\mathrm{T}}$ of the periodic structure is derived as 


$$
\left[\begin{array}{c}
v_{3} \\
\sigma_{23}
\end{array}\right]_{2 N+1}=M^{\mathrm{T}}\left[\begin{array}{c}
v_{3} \\
\sigma_{23}
\end{array}\right]_{1}, M^{\mathrm{T}}=\left(M^{\mathrm{B}} M^{\mathrm{A}}\right)^{N}=\left[\begin{array}{ll}
m_{11}^{\mathrm{T}} & m_{12}^{\mathrm{T}} \\
m_{21}^{\mathrm{T}} & m_{22}^{\mathrm{T}}
\end{array}\right] .
$$

After combining Eqs.(32) and (29), the transmission coefficients can be derived as

$$
\xi=\frac{Z^{2} m_{12}^{\mathrm{T}}+Z\left(m_{11}^{\mathrm{T}}-m_{22}^{\mathrm{T}}\right)-m_{21}^{\mathrm{T}}}{Z^{2} m_{12}^{\mathrm{T}}-Z\left(m_{11}^{\mathrm{T}}+m_{22}^{\mathrm{T}}\right)+m_{21}^{\mathrm{T}}}, \quad \vartheta=\frac{2 Z\left(m_{12}^{\mathrm{T}} m_{21}^{\mathrm{T}}-m_{11}^{\mathrm{T}} m_{22}^{\mathrm{T}}\right)}{Z^{2} m_{12}^{\mathrm{T}}-Z\left(m_{11}^{\mathrm{T}}+m_{22}^{\mathrm{T}}\right)+m_{21}^{\mathrm{T}}}, \quad Z=\rho_{\mathrm{I}}\left(c_{s h}^{\mathrm{I}}\right)^{2} s_{y}^{\mathrm{I}} .
$$

In terms of the problem depicted in Fig. 1(a), Eqs. (27) and (28) are still sufficient to describe the displacement and the magnetic potential function for layers I and II. Therefore, the similar procedures could be applied to calculate the transmission coefficients using Eqs. (29)-(33).

\section{NUMERICAL RESULTS AND DISCUSSION}

In order to validate the theoretical solutions of the dispersion equation and transmission coefficients derived in the previous sections, the elastic properties of polythene and the PM material of Terfenol-D are chosen for the numerical calculation. Their physical parameters are given in Table I as below.

TABLE 1. Material properties of Terfenol-D and polythene.

\begin{tabular}{ccccc}
\hline \hline Properties & $c_{44,} c_{55}\left(10^{9} \mathrm{~N} / \mathrm{m}^{2}\right)$ & $\rho\left(\mathrm{kg} / \mathrm{m}^{3}\right)$ & $h_{15,} h_{24}(\mathrm{~N} / \mathrm{Am})$ & $\mu_{11,} \mu_{22}\left(10^{-6} \mathrm{Ns}^{2} / \mathrm{c}^{2}\right)$ \\
\hline Polythene & 1.28 & 1180 & - & 1.26 \\
Terfenol-D & 15.87 & 9100 & 166.29 & 2.865 \\
\hline \hline
\end{tabular}

${ }^{\mathrm{a}}$ Reference [22]

In the next section, the numerical results for the single layer are firstly calculated and analyzed, and those for the corresponding periodic structures are then presented.

\subsection{Single PM layer}

For sake of easier understanding, the SH wave propagation in the single PM layer (Fig. 1(a)) is considered in this subsection. The SH wave dispersion curves are calculated when the piezomagnetism is ignored, or the magnetic circuit is closed and open. As shown in Fig. 2, the vertical axis corresponds to the slowness $s_{x}$ of the SH wave along $x$-direction, where as the horizontal axis corresponds to the frequency-thickness product $f h$ and normalized wavelength $h / \lambda_{e}$ by $h / \lambda_{e}=f h / c_{s h}^{\mathrm{B}}$.Here $\lambda_{e}$ is the wavelength when the piezomagnetism is ignored, and $\lambda_{e}=c_{s h}^{\mathrm{B}} / f$. First, one needs getting insight on the dispersion curves of the PM plate when the piezomagnetism is ignored (dotted dashed lines in Fig. 2). These curves are calculated using Eq. (16) with the piezomagnetism ignored elastic constant $\mathrm{c}_{44}$. From top to bottom, these curves correspond respectively to different SH modes with the mode order numbered $m$ from 0 to 5 . The zero-order SH mode $(m=0)$ is not dispersive with a constant maximum slowness of the bulk SH wave $\sqrt{\rho / c_{44}}$. It may be regarded as a SH skimming wave along $x$-axis with no wave penetration in the thickness of the plate. Every higher order SH mode $(m>0)$ is dispersive with a cut-off frequency or wavelength. The cut-off wavelength is found to be $2 h / m$, i.e. the ratio of twice the thickness $h$ to the mode number $m$. These higher SH wave modes approach the zero-order mode in the high frequency or small wavelength limit with the same physical nature.

The dispersion curves of the magnetically closed PM plate (thick solid lines in Fig. 2 ) calculated using Eq. (16), have the same feature as that of the piezomagnetism ignored situation described in the preceding paragraph with the cut-off frequency shifted to the higher value domain and the maximum slowness to the smaller value determined by the piezomagnetic-stiffened elastic constant $\hat{\mathrm{c}}_{44}\left(\hat{\mathrm{c}}_{44}>\mathrm{c}_{44}\right)$. The dispersion curves for the piezomagnetism ignored or the magnetic closed PM plate well agree with former work [23]. The dispersion curves of the magnetically open PM plate (dashed lines in Fig. 2) calculated using Eq. (18), are of quite similar nature as those determined within the piezomagnetism ignored and magnetically closed situations, except that the zero-order SH mode becomes dispersive in that case. The difference comes from the coupling of the magnetic and elastic fields that introduces the nonzero term $h_{15}$ in Eq. (18). If $h_{15}$ approaches zero, the dispersion curves correspondingly becomes those of the magnetically closed case.

To further reveal the properties of the single layer, the transmissivity or the reflectivity of the SH wave at normal incidence from the Layer $I$ is calculated. The reflectivity curves corresponding to the three cases depicted in Fig. 2 are calculated using Eqs. (29) and (33), in which the incident angle $\theta_{0}$ is set to zero. The same horizontal coordinates and similar curve types are chosen for comparison. As shown in Fig. 3, the reflectivity curve for the piezomagnetism ignored PM plate 
(dotted dashed lines in Fig. 3) goes to zero when the wavelength $\lambda_{e}$ is equal to the ratio of the mode number $m(1,2,3 \ldots)$ to twice the thickness $h$, which corresponds to the $m^{\text {th }}$ order of the SH wave mode at $s_{x}=0$ (dotted dashed lines in Fig. 2). This means that the $\mathrm{SH}$ waves can fully penetrate to the layer II when there is a corresponding solution in the dispersion curve. On the other hand, the SH wave undergoes total reflection if there is no solution in the dispersion curve as the reflectivity $R$ approaches the value of one. Same features can be observed for the reflectivity curves calculated for the magnetically closed (solid lines in Fig. 3) and magnetically open (dashed lines in Fig.3) PM plate.

Let us now consider the reflectivity spectra of the SH wave when it is incident from the layer $I$. The reflective spectra corresponding to the three cases in Fig. 2 are calculated using Eqs. (29) and (33). For further comparisons with experiments, the vertical coordinate is set to be the incident angle $\theta_{0}$ instead of the slowness. This parameter can be switched to the slowness $s_{x}$ by $s_{x}=\sin \theta_{0} / c_{s h}^{\mathrm{A}}$. As shown in Fig. 4(a), the reflectivity of the piezomagnetism ignored PM plate goes to zero when there is a corresponding solution in the dispersion curve; the reflectivity approaches one if there is no solution in the dispersion curve. The dark lines (Fig. 4(a)) of various SH modes in the spectra are identical to the corresponding dispersion curves (dotted dashed lines in Fig 2). The zero-order SH wave is excited when the incident angle reaches the critical angle $52^{\circ}$, as shown in Fig. 4(a).

Same features can be observed for the reflective spectra calculated for the magnetically closed (Fig. 4(b)) and magnetically open (Fig. 4(c)) conditions, except that the dark lines at high incident angle in Fig. 4(b) is slightly small in the spectra to the corresponding dispersion curves (solid lines in Fig. 2) for the influence of the magnetic permeability of the layer $I$. Since the occurrence of piezomagnetism increases the shear velocity of the PM material, the fundamental cut-off frequency or wavelength is different from the case where the piezomagnetism is ignored. The fundamental cut-off wavelength $\lambda_{e}$ in Fig. 4(a) must be replaced by the piezomagnetism increased wavelength $\lambda_{p}\left(\lambda_{p}=\bar{c}_{s h}^{\mathrm{B}} / f\right)$ as shown in Figs. 4(b) and (c). For the magnetically closed PM plate (Fig. 4(b)), the cut-off wavelength is found to be $2 h / m$. For the magnetically open PM plate (Fig. 4(c)), the cut-off wavelength is found to be $2 \mathrm{~h} / \mathrm{m}$ for even number modes, and slightly below $2 \mathrm{~h} / \mathrm{m}$ for the odd number modes. The mismatch is inversely proportional to the frequency-thickness product when $m$ is odd. The zero-order SH wave is excited when the incident angle reaches the critical angles $39.5^{\circ}$ or $42^{\circ}$ as shown in Fig. 4 (b) Fig. 4(c) respectively.

\subsection{Periodic structure}

The comparison of the corresponding dispersion curves and reflective spectra of a single PM layer when the piezomagnetism is ignored, the magnetic circuit being closed or open, have clearly revealed the complex behavior of the SH wave propagation. In this subsection, we show that even more complicated phenomena happen when one considers the periodically-layered structures. To calculate the band gaps and the transmission coefficients for the corresponding situations, we have considered a periodic structure featuring 20 unit cells, as shown in Fig. 1(b). The thickness of the elastic layer A is assumed to be $h_{A}=c_{s h}^{\mathrm{A}} /\left(4 f_{0}\right)$ where the bulk shear velocity is $c_{s h}^{\mathrm{A}}=\sqrt{c_{44}^{\mathrm{A}} / \rho_{\mathrm{A}}}$; the thickness of the PM layer $\mathrm{B}$ is assumed to be $h_{B}=\bar{c}_{s h}^{\mathrm{B}} /\left(4 f_{0}\right)$, and it becomes $c_{s h}^{\mathrm{B}} /\left(4 f_{0}\right)$ if the piezomagnetism is ignored.

As already done in the section devoted to the single PM plate, the normal incidence is firstly considered for the periodic structure. Band gaps of the periodic structure are calculated using Eqs. (22) and (26). As shown in Fig. 5, the horizontal coordinate is the normalized periodic wave number $\Lambda d / \pi$ and the vertical coordinate is the normalized angular frequency $\omega / \omega_{e}$. Here $\omega_{e}$ is the primary angular frequency of the periodic structure when the layer B is exempt of piezomagnetism, and $\omega_{e}=\pi /\left(h_{\mathrm{A}} / c_{s h}^{\mathrm{A}}+h_{\mathrm{B}} / c_{s h}^{\mathrm{B}}\right)$. When the piezomagnetism accounted for, the primary angular frequency is identified as $\omega_{p}$ instead of $\omega_{e}$, and $\omega_{p}=\pi /\left(h_{\mathrm{A}} / c_{s h}^{\mathrm{A}}+h_{\mathrm{B}} / \bar{c}_{s h}^{\mathrm{B}}\right)$.

We first have analyzed the band structures in the case where the piezomagnetism is ignored. As show in Fig. 5(a), stop bands appear regularly when $\omega / \omega_{e}$ is an odd integer while pass bands appear regularly when $\omega / \omega_{e}$ is an even integer. Same features can be observed for the band gaps when the PM layer B is under magnetically closed boundary condition (Fig. 5(b)). The widths of the stop bands are respectively $1.2 \omega_{e}$ and $1.28 \omega_{p}$ for the first two cases. The small difference in the band width is caused by the different ratio of SH wave impedances of the layer B to that of the layer A, which are respectively 9.78 and 12.4. When the magnetic boundary condition changes from the closed circuit to the open circuit (Fig. 5(c)), the band gap changes in a large amount, from $\omega / \omega_{p}=1.04$ to 1.39 .

We have also calculated the transmissivity of three corresponding cases in Fig.5 for potential experimental verification. As shown in Fig. 6(a) for the piezomagnetism ignored case, the stop bands open up when the transmissivity $T$ is zero, whereas a pass band appears when the transmissivity $T$ is different from zero. The band structures are identical to those 
calculated by the dispersion equation (Fig. 5(a)). Similar features can be found for the magnetically closed (Figs. 5(b) and 6(b)) and open cases (Figs. 5(c) and 6(c)).

As we did for the single PM plate, we now consider the case of the periodic structure excited at oblique incidence. The band gaps of three corresponding cases to the ones depicted in Fig. 5 are determined using Eqs. (22) and (26). We first analyze the band structure of the periodic stacking when the piezomagnetism is ignored (see Fig. 7(a)). The vertical coordinate is for the incident angle $\theta_{0}$, and the horizontal coordinates correspond respectively the frequency-thickness product $f h_{\mathrm{B}}$ and the normalized angular frequency $\omega / \omega_{e}$. As noticed by Fin. Fig.7(a), the pass bands (shown in black color) exist when $\omega / \omega_{e}$ is even with $\theta_{0}$ being zero, while the stop bands (shown in white color) exist when $\omega / \omega_{e}$ is odd. This is in good agreement with the corresponding results of the normal incidence. As $\theta_{0}$ increases, new stop bands emerge from the original pass zones except for the first-order pass band. When the incident angle is above the critical angle of $52^{\circ}$, the $y$ coordinate displacement component of the wave in the layer B shall decay exponentially, since the bulk shear wave velocity of the polythene is smaller than that of the Terfenol-D.

Similar features can be observed when the PM layer B of the periodic structure is magnetically closed (see Fig. 7(b)) or open (see Fig. 7(c)). As shown in Fig. 7(c), affected by the magnetically open circuit, the normalized frequency does not strictly take the feature as that shown in Fig. 7(a). Small stop bands arise when $\omega / \omega_{p}$ is close to 2 or 6 with $\theta_{0}$ being zero. The critical angle of the three cases can be taken the same as their corresponding ones, and are respectively $52^{\circ}, 39.5^{\circ}$ and $42^{\circ}$.

We have also calculated the reflective spectra of the three corresponding cases depicted in Fig. 7 for potential experimental verification. The situation when the piezomagnetism is ignored (Fig. 8(a)) is first analyzed. The stop bands exist where the reflectivity is equals to unity, and these stop bands (Fig. 8(a)) are in good agreement with those derived from the dispersion relation (Fig. 7(a)). Same features are found for the magnetically closed (Figs. 7(b) and 8(b)) or open cases (Figs. $7(\mathrm{c})$ and $8(\mathrm{c}))$.

\section{CONCLUSIONS}

In this article, the propagation of SH wave in a single PM layer and in a periodically-layered PM structure is studied. Theoretical solution, numerical calculation and analysis allowed drawing the following conclusions:

In terms of the dispersion curves in a single plate when the piezomagnetism is ignored, the zero-order SH mode is found to be not dispersive with a maximum slowness, while any higher order SH mode is dispersive with a cut-off wavelength of the division of mode number $m$ to be twice the plate thickness. Identical features are found for the dispersion curves of the magnetically closed and open situations, except that the cut-off frequency of the former case shifts to a relatively high value and the zero-order SH mode of the latter case is gets dispersive. The SH wave can fully penetrate the single PM layer when there is a corresponding solution in the dispersion curve; in contrast, it is fully reflected if there is no solution in the dispersion curve, the piezomagnetism being ignored, or the magnetic circuit being closed or open.

In the case of the periodically-layered PM structure, and when the piezomagnetism is ignored, stop bands appear if the normalized frequency is an odd integer for the normal incidence; , correspondingly, pass bands cover the reverse areas. New stop bands open up where the pass bands exist and separate them. Similar feature are observed for the band gaps of the magnetically closed and open situations, except that the critical angle in the former case becomes smaller and the zero-order SH mode in the latter case is dispersive. In the case where the piezomagnetism is ignored, or where the magnetic circuit is closed or open, the stop bands of the periodic structure change from zero to a maximum. On the other hand it goes to zero as the geometric ratio ranges from zero to unity. Besides, the stop bands repeat such a cycle as the normalized frequency increases under the corresponding critical angles.

The above theoretical findings may help designing phononic crystals for potential applications, and therefore corresponding experimental verifications are required to realize the expected applications. The corresponding wave propagation for a twodimensional case will be more meaningful and practical, and the extension to the two-dimensional case is going to be considered.

\section{ACKNOWLEDGMENTS}

This work was supported by the Natural Science Foundation of China under Grant Nos. under 11090333 and 11090334.

\section{REFERENCES}

[1] M. S. Kushwaha, P. Halevi, L. Dobrzynski, and B. Djafari-Rouhani, Acoustic band structure of periodic elastic composites, Phys. Rev. Lett. 71 (1993) 2022-2025.

[2] D. García-Pablos, M. Sigalas, F. R. Montero de Espinosa, M. Torres, M. Kafesaki, and N. García, Theory and 
LIU, ZHAO, PAN, BONELLO, ZHONG

Experiments on Elastic Band Gaps, Phys. Rev. Lett. 84 (2000) 4349-4352.

[3] R. Esquivel-Sirvent and G. H. Cocoletzi, Band structure for the propagation of elastic waves in super lattices, J. Acoust. Soc. Am. 95 (1994) 86-90.

[4] L. Wang and S. I. Rokhlin, Floquet wave homogenization of periodic anisotropic media, J. Acoust. Soc. Am. 112 (2002) $38-45$.

[5] A. Baz, Active control of periodic structures, J. Vib. Acoust. 123 (2001) 472-479.

[6] Z. Qian, F. Jin, Wang Z., and K. Kishimoto, Dispersion relations for SH-wave propagation in periodic piezoelectric composite layered structures, Int. J. Eng. Sci. 42 (2004) 673-689.

[7] J. Liu, W. Wei, and D. Fang, Propagation behavior of shear horizontal waves in peizoelectric-piezomagnetic periodically layered structures, Acta. Mech. Solida. Sin. 23 (2010) 77-84.

[8] Y. Wang, F. Li, W. Huang, X. Jiang, Y. Wang, and K. Kishimoto, Wave band gaps in two-dimensional piezoelectric/piezomagnetic phononic crystals, Int. J. Solids. Struct. 45 (2008) 4203-4210.

[9] Y. Pang, Y. Wang, J. Liu, and D. Fang, A study of the band structures of elastic wave propagating in piezoelectric/piezomagnetic layered periodic structures, Smart. Mater. Struct. 19 (2010) 055012-1-055012-16.

[10] J. Zhao, Y. Pan, and Z. Zhong, Theoretical study of shear horizontal wave propagation in periodically layered piezoelectric structure, J. Appl. Phys. 111 (2012) 064906-1-064906-11.

[11] M.I.Bichurin, V.M.Petrov, R.V.Petrov, Direct and inverse magnetoelectric effect in layered composites in electromechanical resonance range: A review, J. Magnetism and Magnetic Mater. 324 (2012) 3548-3550.

[12] L.Y.Fetisov, D.V. Chashin, Y. K. Fetisov, A. G. Segalla, G. Srinivasan, Resonance magnetoelectric effects in a layered composite under magnetic and electrical excitations, J. Appl. Phys. 112 (2012) 014103-1 - 014103-6.

[13] Y.Z. Wang, F.M. Li, K.Kishimoto, Y.S. Wang, W.H. Huang, Elastic wave band gaps in magnetoelectroelastic phononic crystals, Wave Motion. 45 (2009) 47-56.

[14] B. A. Auld, D. E. Chimenti, and P. J. Shull, Shear horizontal wave propagation in periodically layered composites, IEEE. T. Ultrason. Ferr. 43 (1996) 319-325.

[15] G.Q. Nie, J. X. Liu, Z. J. An, X. F. Zhao, Shear horizontal wave propagation in two layered piezoelectric/piezomagnetic coupled plates, International Conference on Smart Materials and Nanotechnology in Engineering 6423 (2007) 64235T-1-

64235T-7.

[16] F. Li and Y. Wang, Study on wave localization in disordered periodic layered piezoelectric composite structures, Int. J. Solids. Struct. 42 (2005) 6457-6474.

[17] F. Li, M. Xu, and Y. Wang, Frequency-dependent localization length of SH-wave in randomly disordered piezomagnetic phononic crystals, Solid. State. Commun. 141 (2007) 296-301.

[18] J. A. Otero, H. Calás,R. Rodríguez-Ramos, G. A. Maugin, G. Monsiváis, and R. Pérez-Alvarez, Diffraction of transverse horizontal waves in Fibonacci piezoelectric super lattices, Z. Angew. Math. Phys. 55 (2004) 519-533.

[19] G..Q. Nie, J. X. Liu, Z. J. An, Elastic wave propagation in piezoelectric/piezomagnetic bi-material plates, Eng. Mech. 27 (2010) 30-36.

[20] J. Chen, E. Pan, and H. Chen, Wave propagation in magneto-electro-elastic multilayered plates, Int. J. Solids. Struct. 44 (2007) 1073-1085.

[21] S. I. Rokhlin and L. Wang, Stable recursive algorithm for elastic wave propagation in layered anisotropic media: Stiffness matrix method, J. Acoust. Soc. Am. 112 (2002) 822-834.

[22] Soon Suck Jarng, Magnetostrictive Terfenol-D material linear simulation using element method, Inter. J. Appl. Electro. Mech. 24 (2006) 187-193.

[23] Calas, H., J. A. Otero, R. Rodriguez-Ramos, G. Monsivais and C. Stern. Dispersion Relations for Sh Wave in MagnetoElectro-Elastic Heterostructures, Int. J. Solids Struct. 45 (2008), 5356-5367. 


\section{Figure Captions}

FIG. 1. Schematic diagram of SH wave propagating through (a) a single layer and (b) a periodic structure.

FIG. 2. SH wave dispersion curves of the single PM layer when (1) piezomagnetism ignored (dot dashed lines), (2) magnetic closed (thick solid lines) and (3) magnetic open (dashed thick lines).

FIG. 3. Reflectivity of the normal incident wave for the single PM layer when (1) piezomagnetism ignored (dot dashed lines), (2) magnetic closed (solid lines) and (3) magnetic open (dashed lines).

FIG. 4(a). Reflective spectrum of the incident wave for the single PM layer when piezomagnetism ignored.

FIG. 4(b). Reflective spectrum of the incident wave for the single PM layer when magnetic closed.

FIG. 4(c). Reflective spectrum of the incident wave for the single PM layer when magnetic open.

FIG. 5. Band structures of the normal incident wave for the periodic structure when the PM layer B (a) ignored piezomagnetism, (b) magnetic closed, and (c) magnetic open.

FIG. 6. Transmissivities of the normal incident wave for the periodic structure when the PM layer B (a) ignored piezomagnetism, (b) magnetic closed, and (c) magnetic open.

FIG. 7(a). Band structures of the incident wave for the periodic structure when the PM layer B ignored piezomagnetism (stop bands in black).

FIG. 7(b). Band structures of the incident wave for the periodic structure when the PM layer B magnetic closed (stop bands in black).

FIG. 7(c). Band structures of the incident wave for the periodic structure when the PM layer B magnetic open (stop bands in black).

FIG. 8(a). Reflective spectrum of the incident wave for the periodic structure when the PM layer B ignored piezomagnetism.

FIG. 8(b). Reflective spectrum of the incident wave for the periodic structure when the PM layer B magnetic closed.

FIG. 8(c). Reflective spectrum of the incident wave for the periodic structure when the PM layer B magnetic open. 
Fig. 1
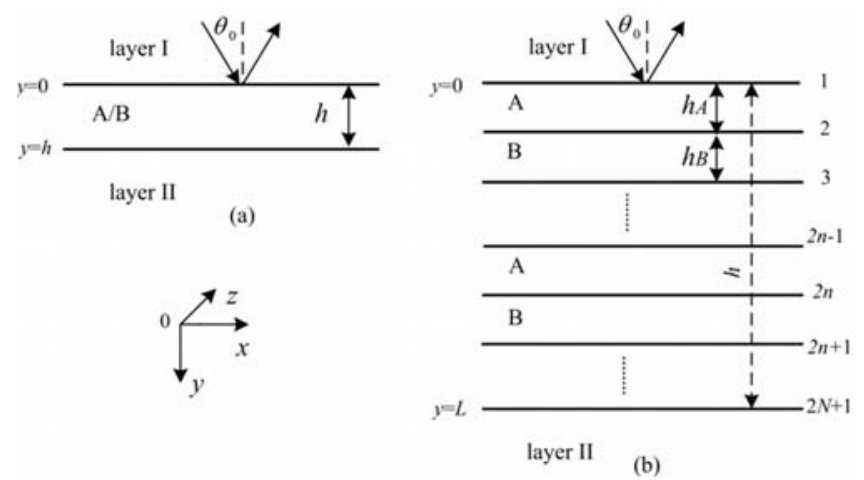

Fig. 2

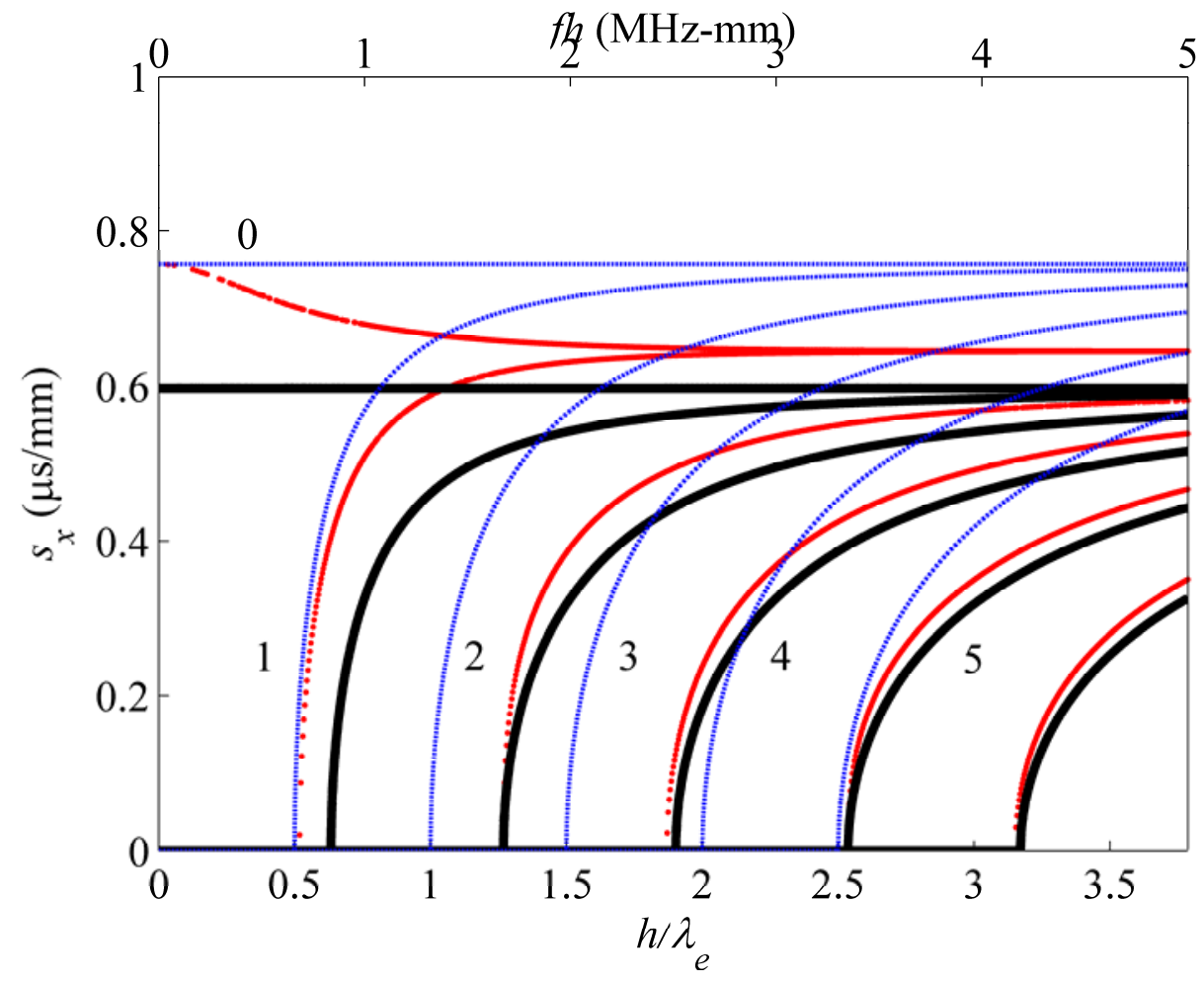


Fig. 3

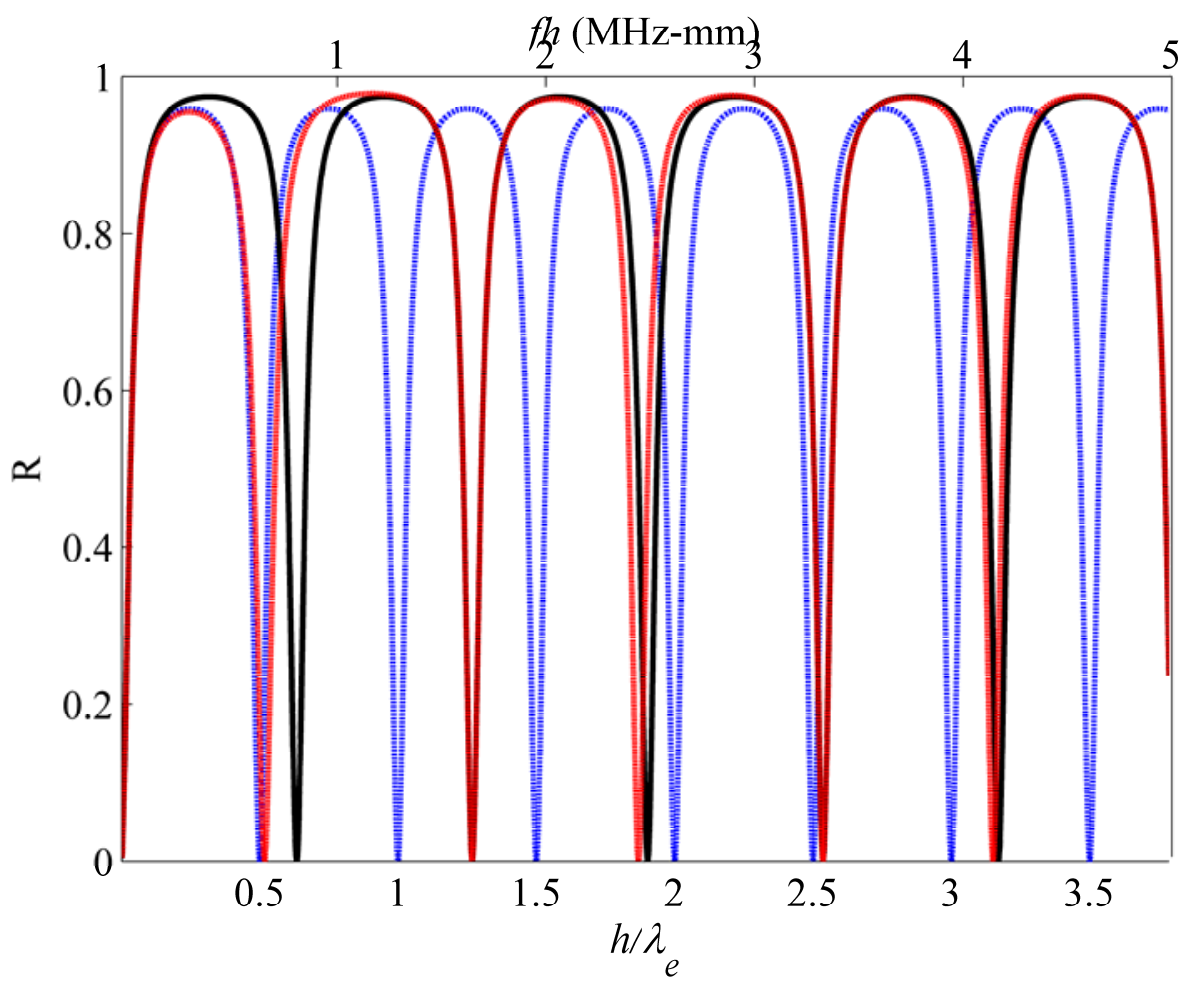

Fig. 4(a)

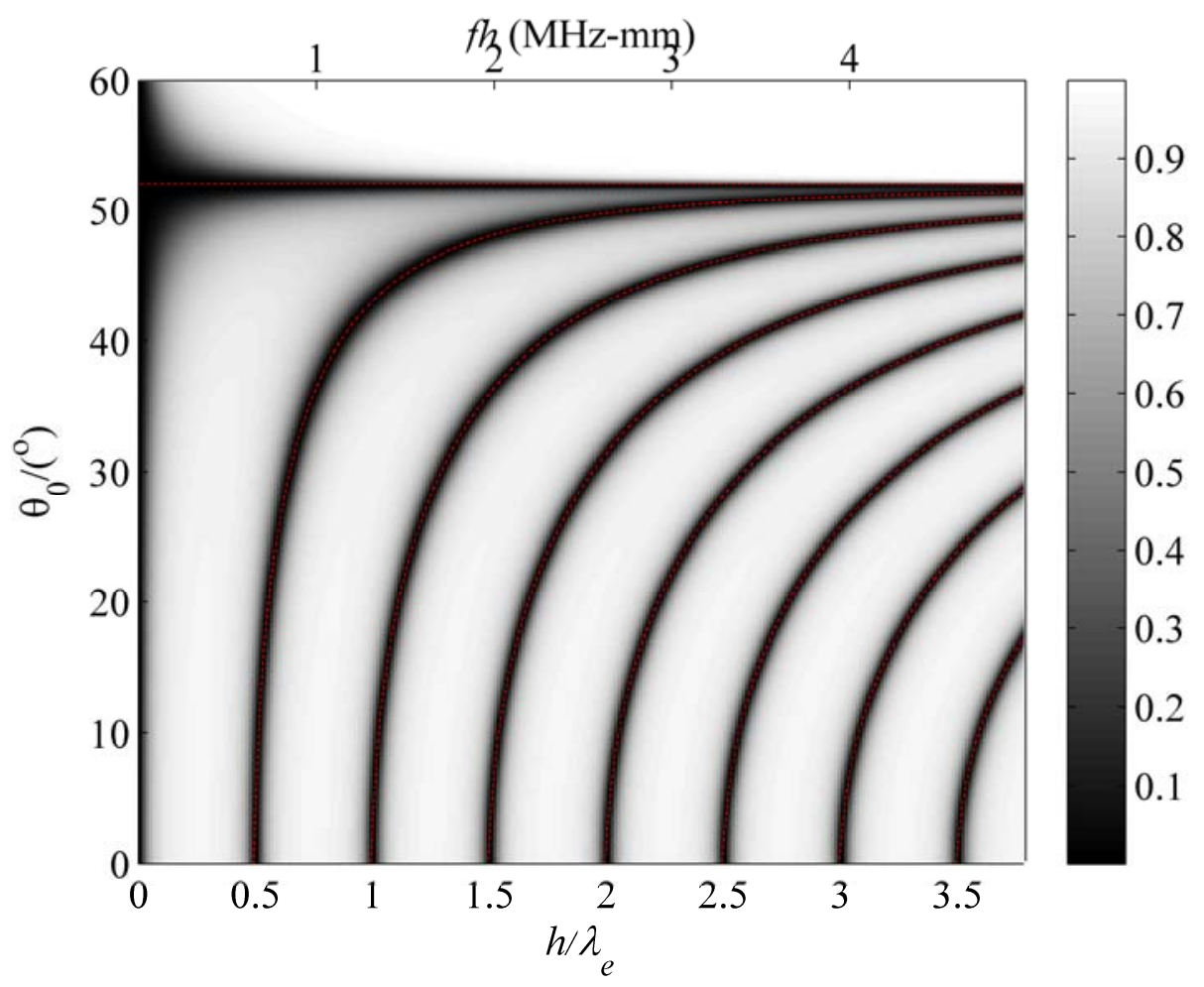


Fig. 4(b)

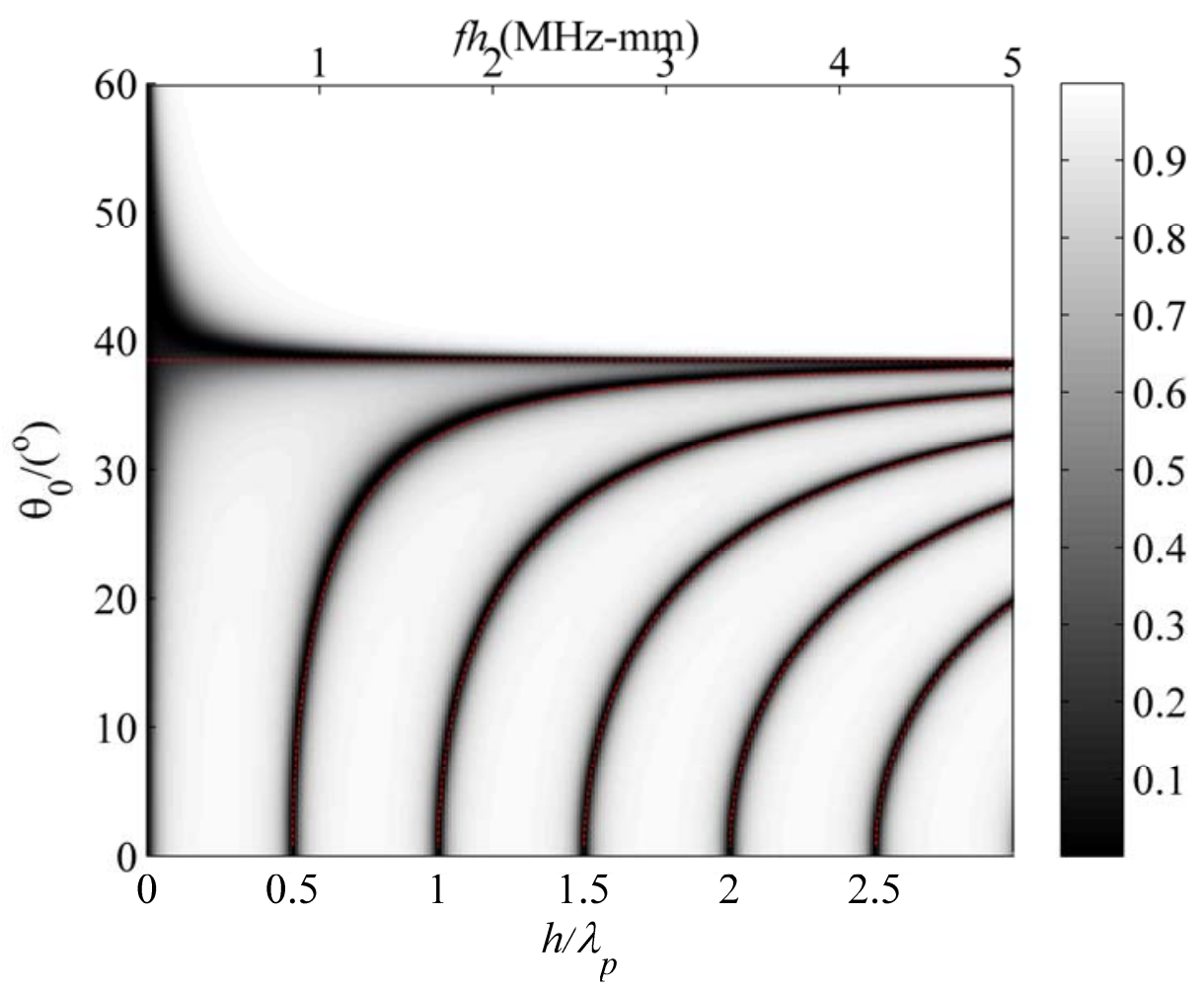

Fig. 4(c)

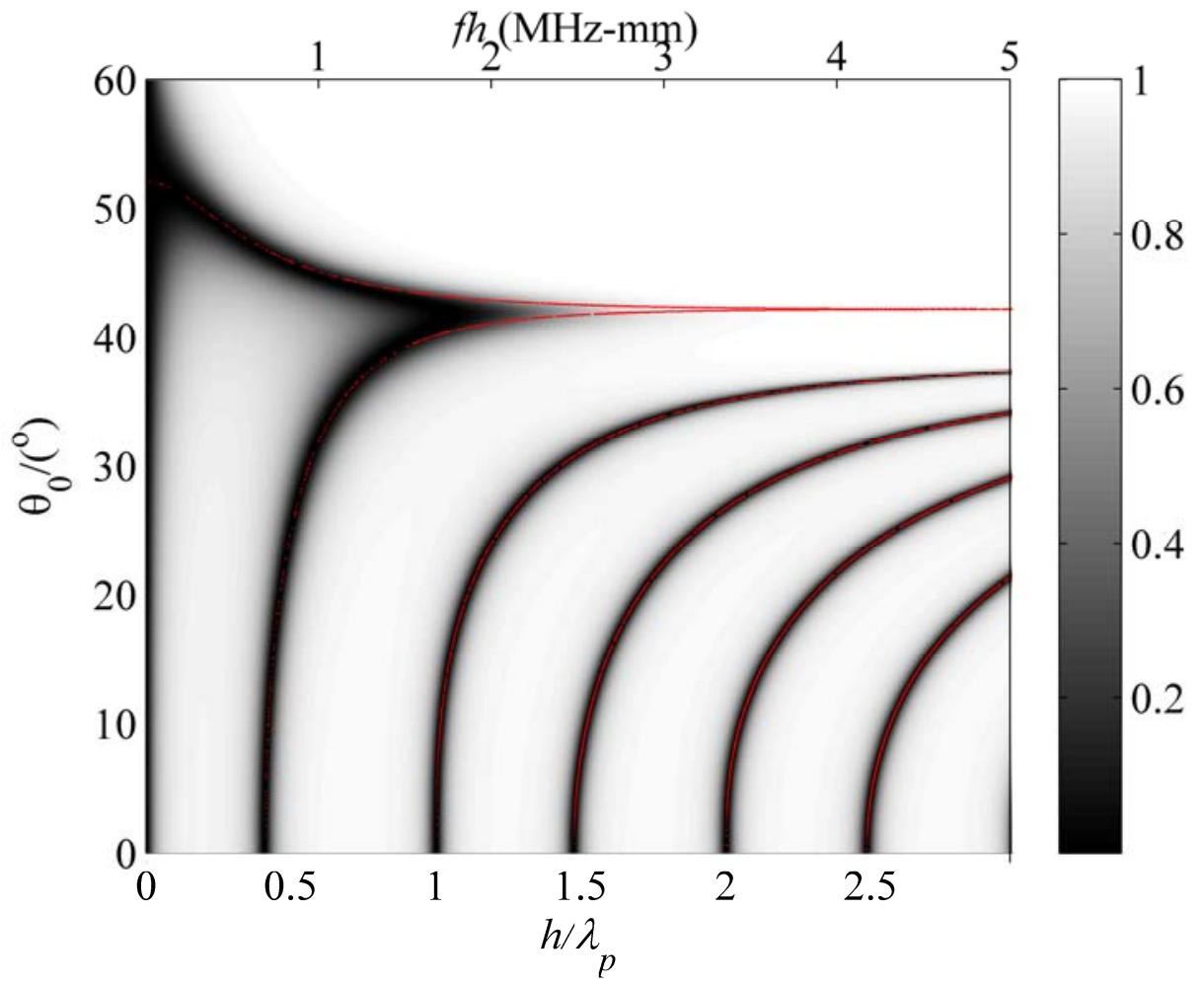


Fig. 5

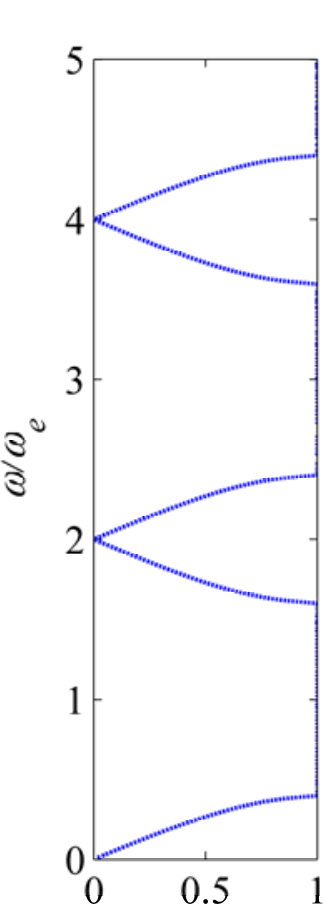

(a)

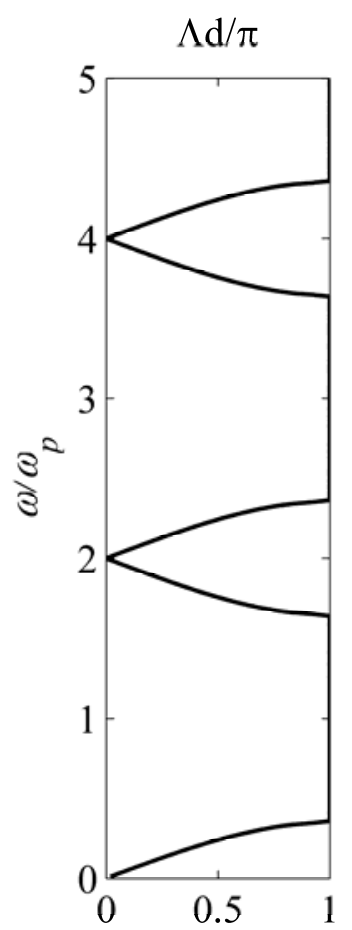

(b)

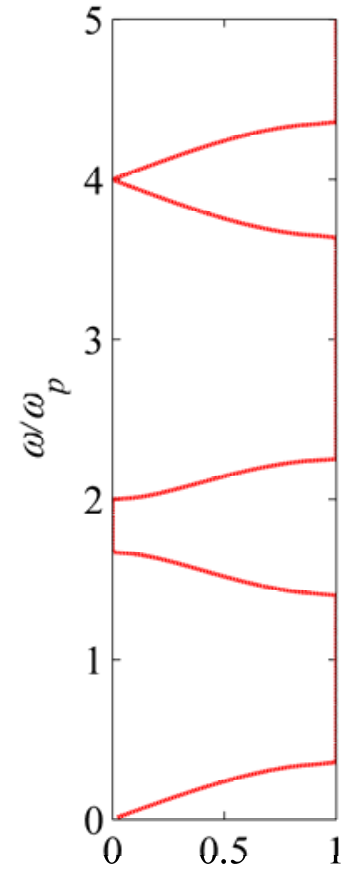

(c)

Fig. 6

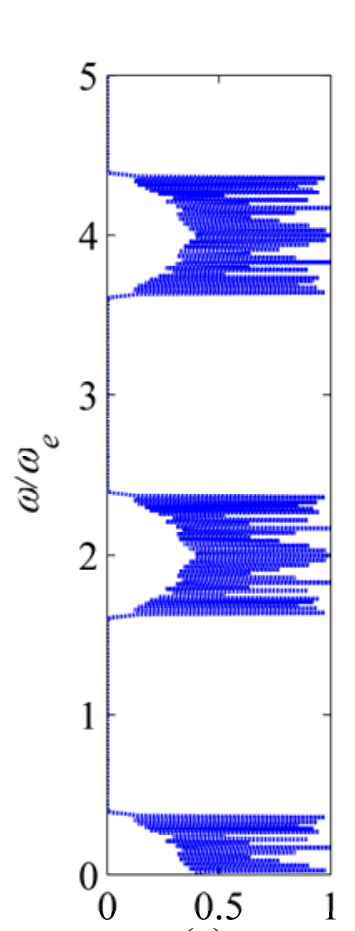

(a)

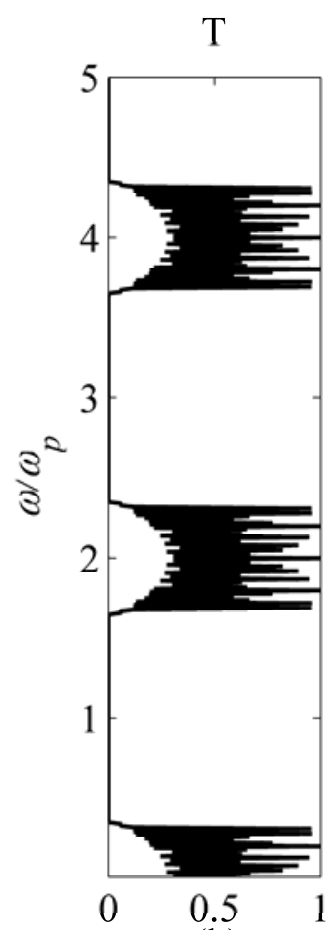

(b)

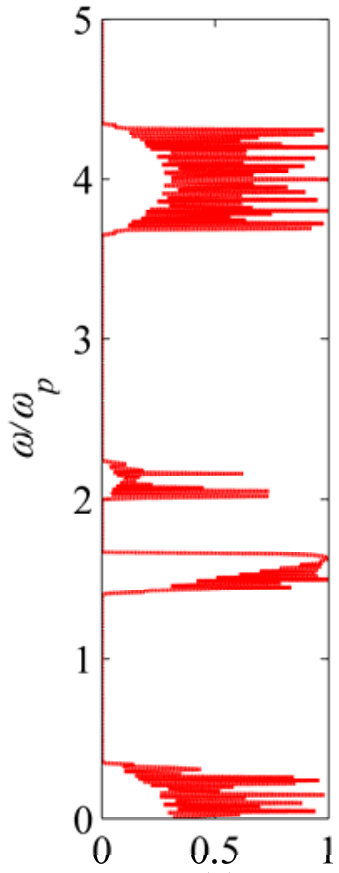

(c) 
Fig. 7(a)

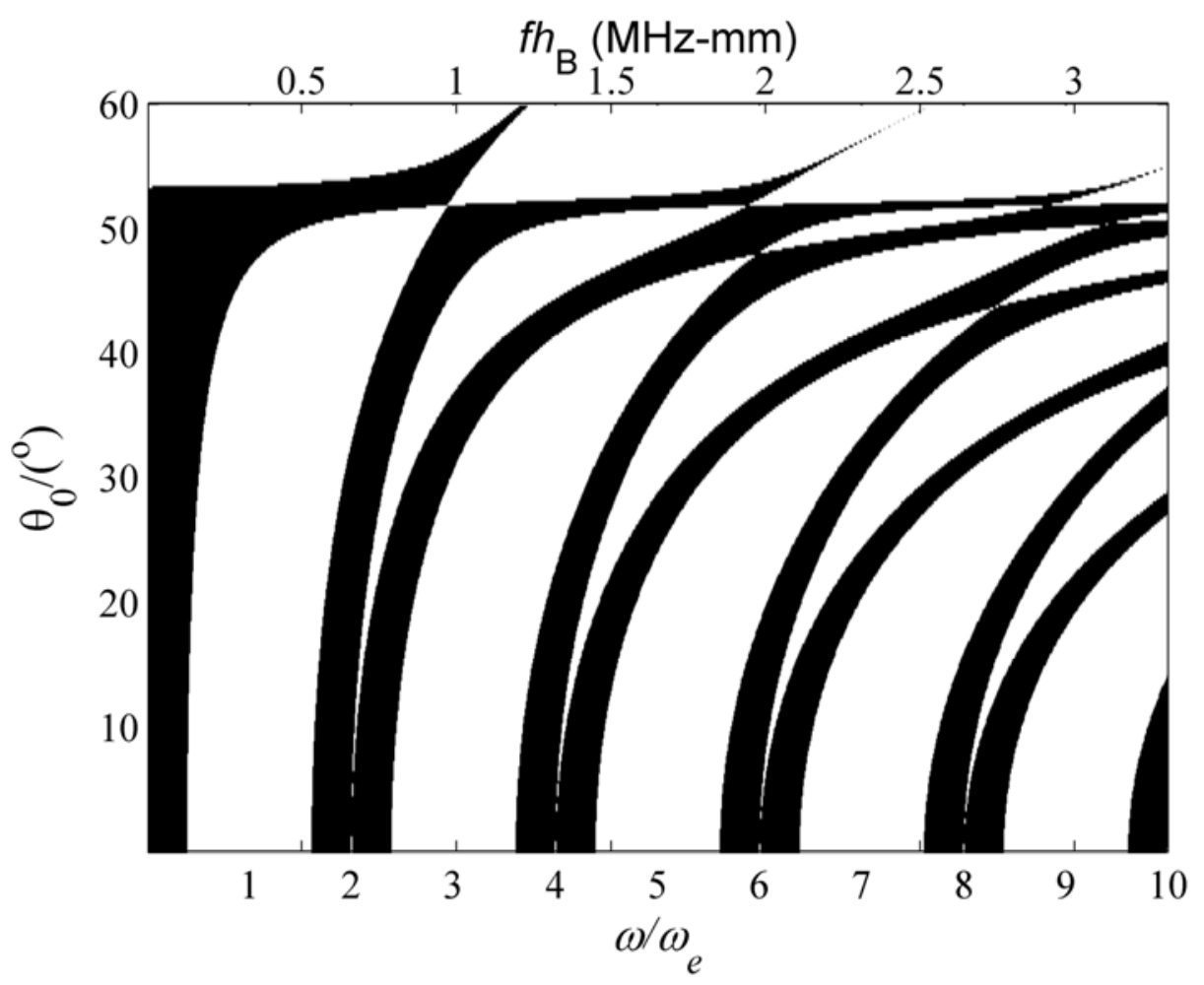

Fig. 7(b)

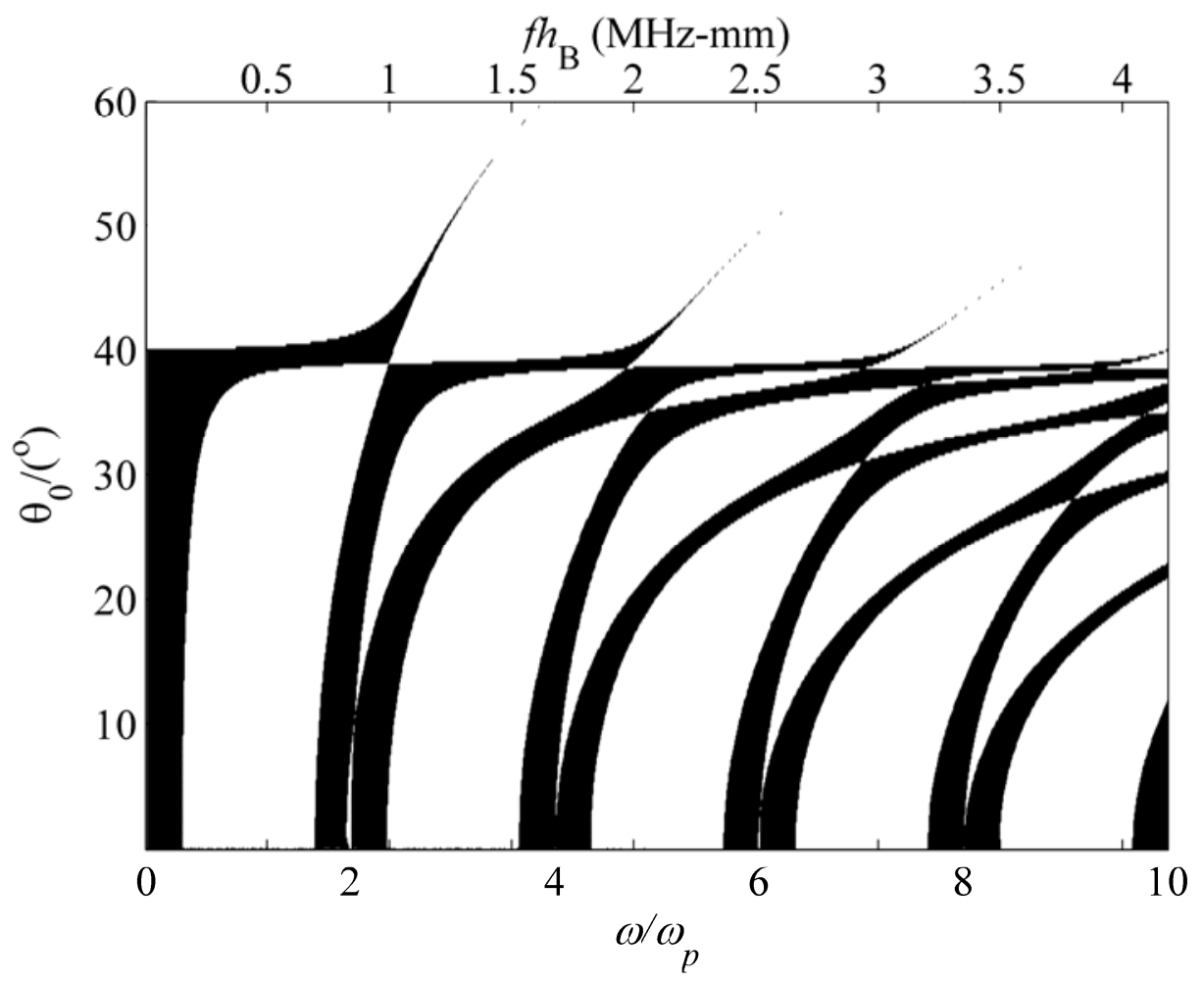


Fig. 7(c)

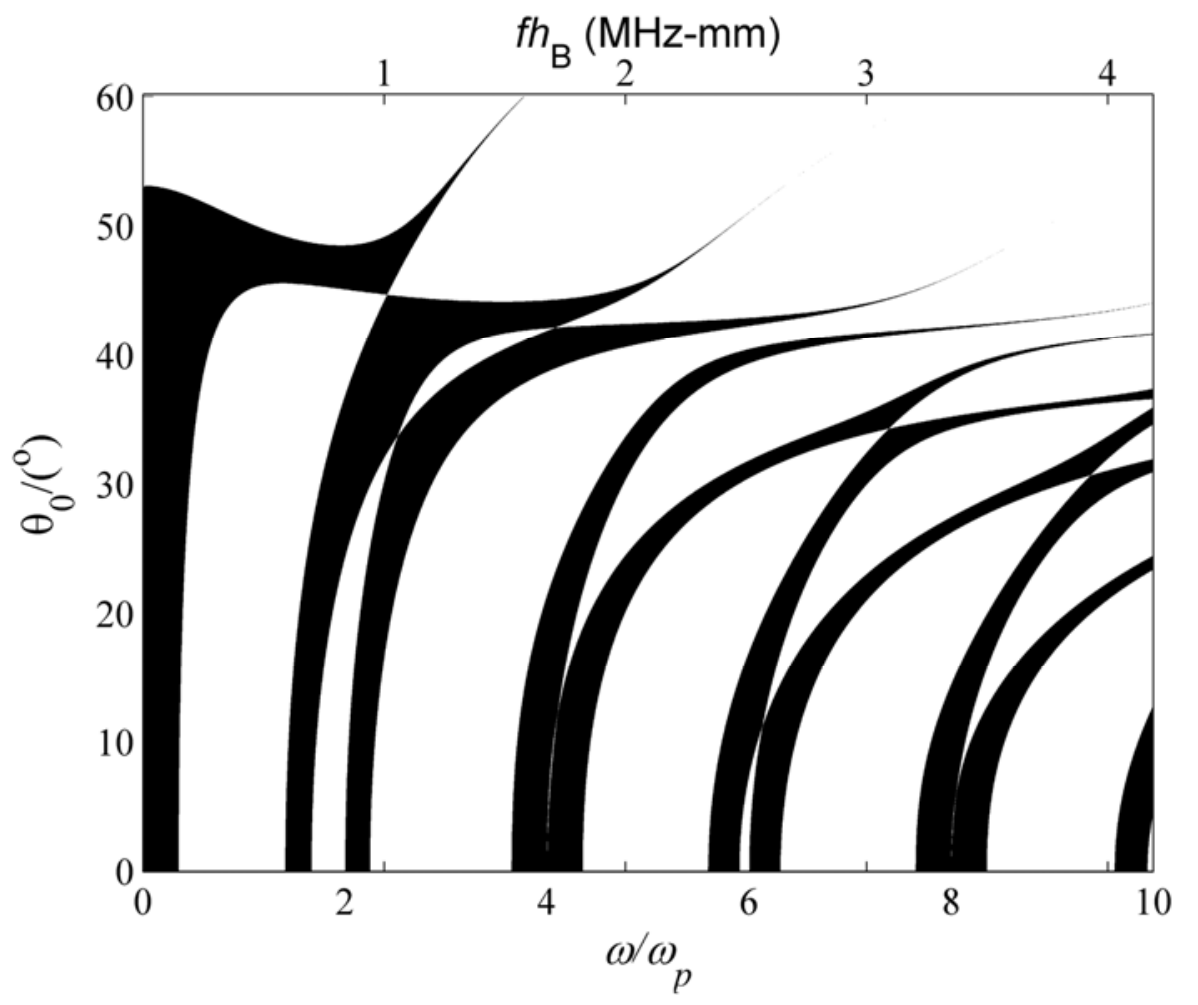

Fig. 8(a)

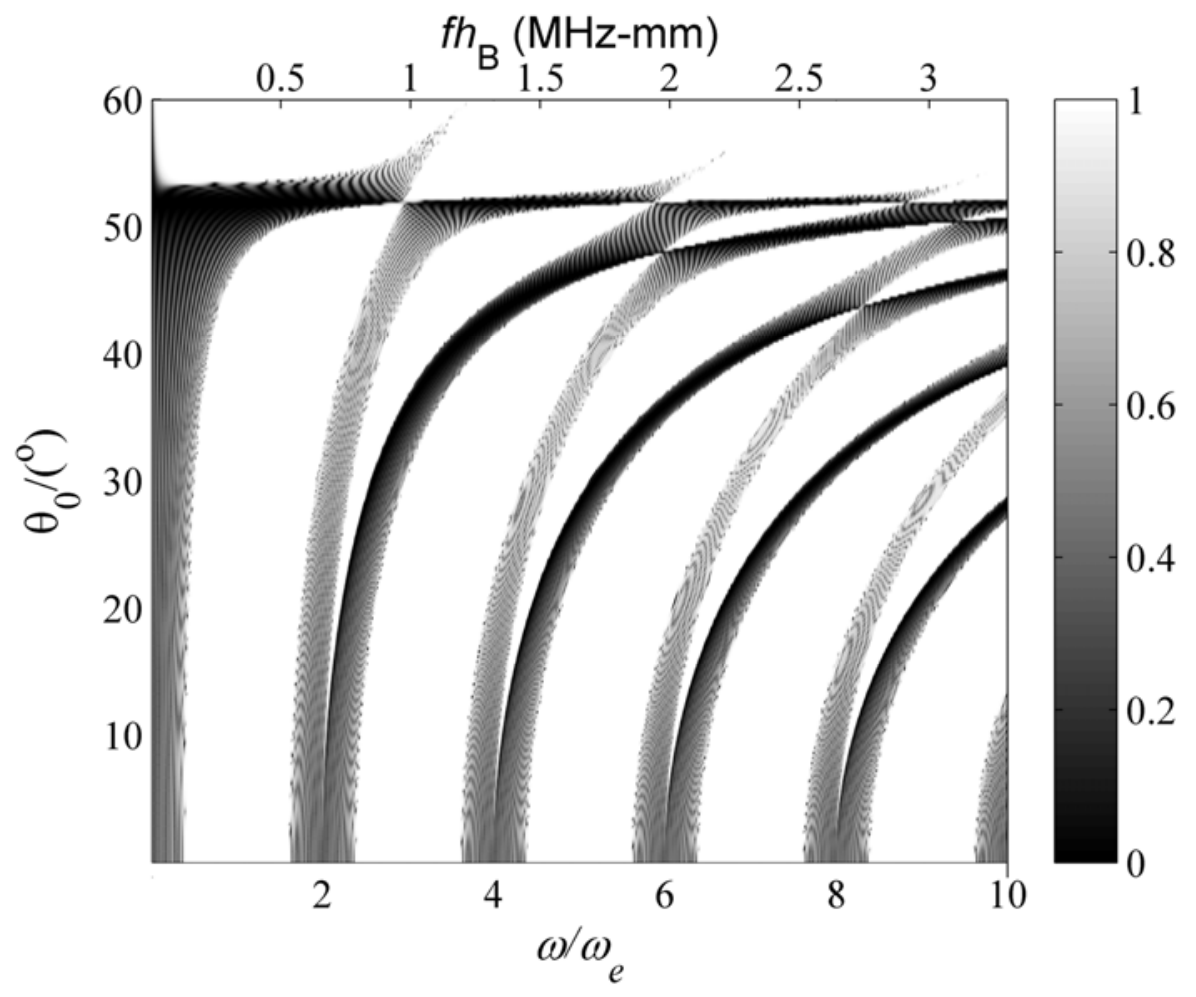


Fig. 8(b)

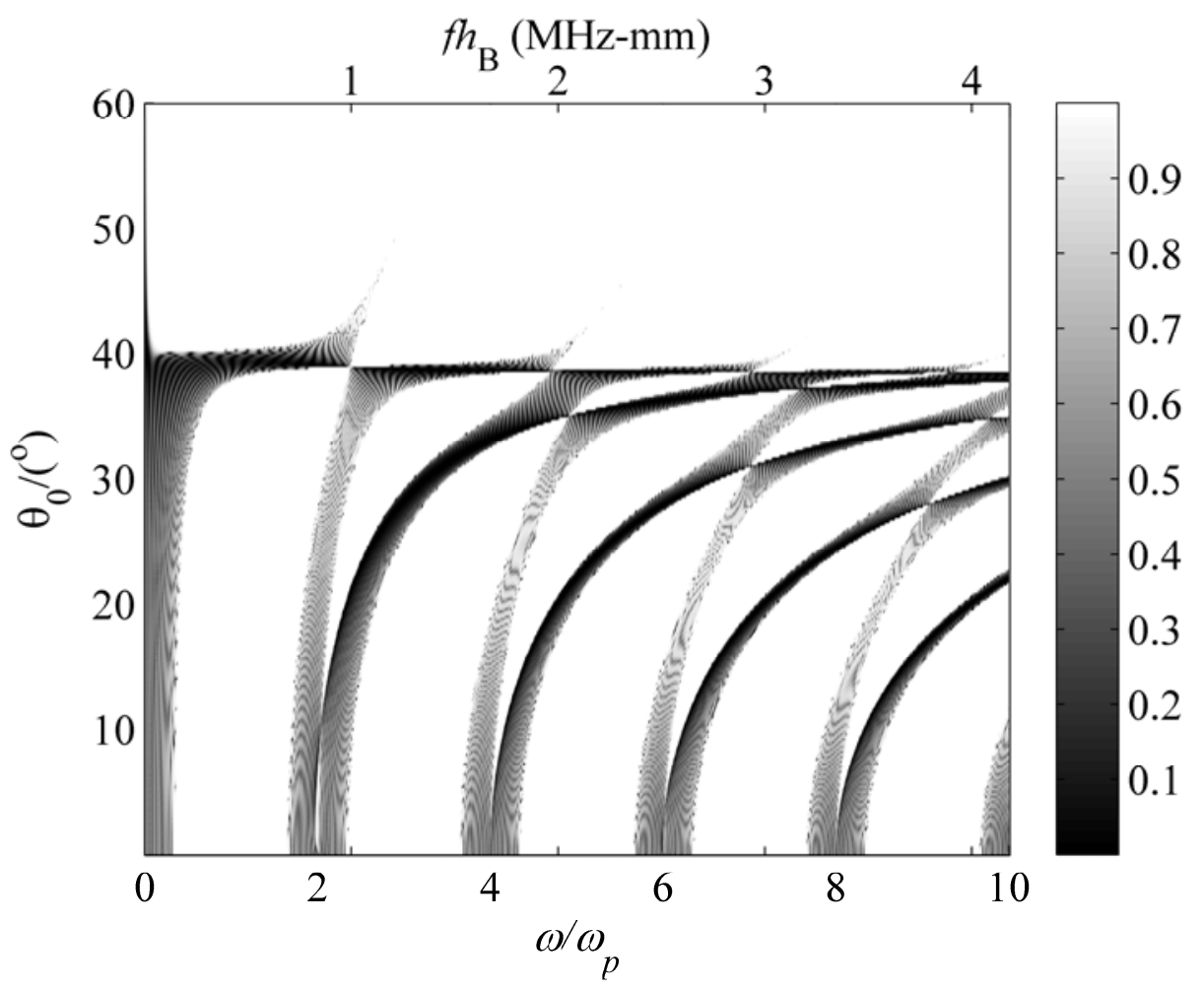

Fig. 8(c)

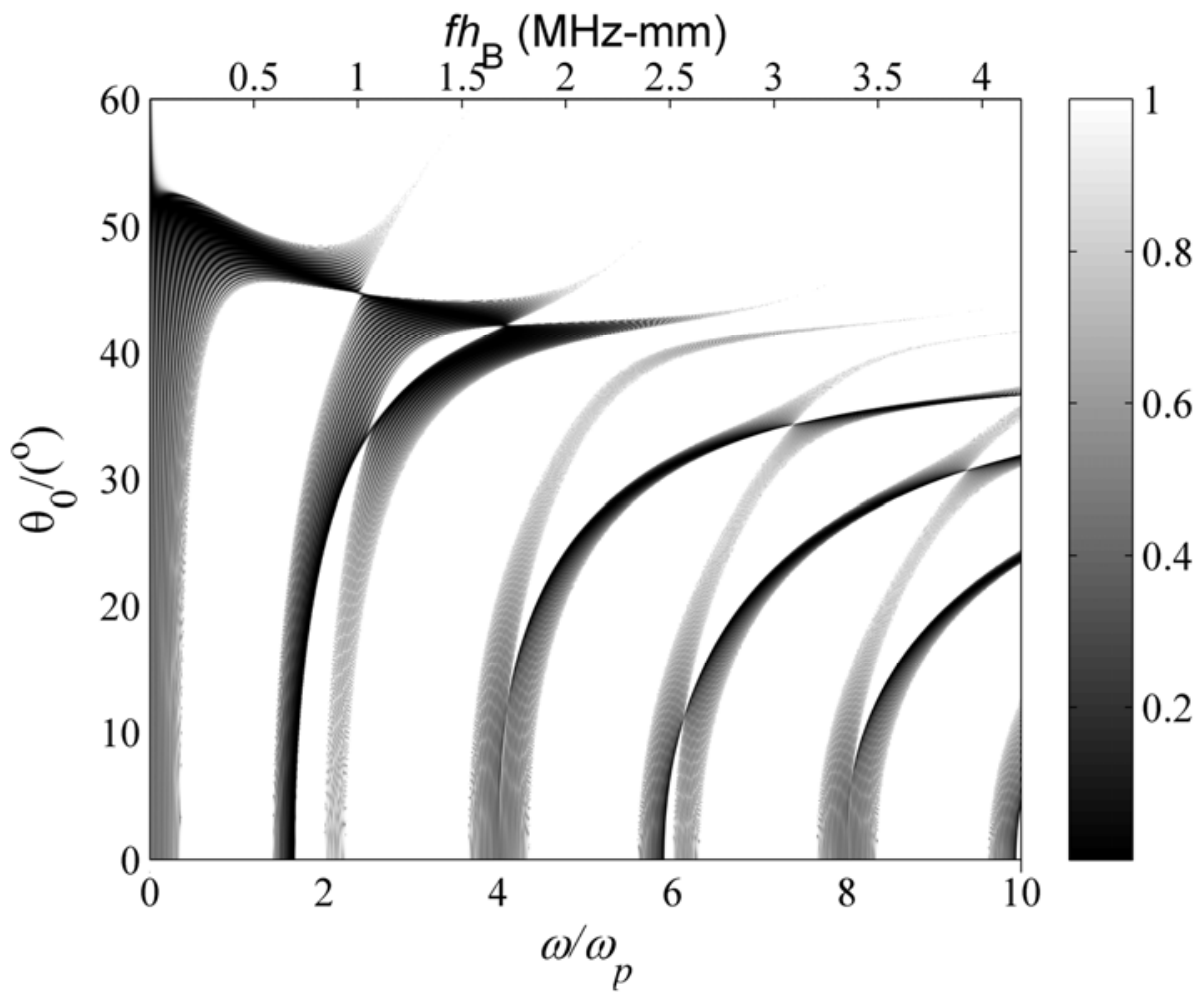

